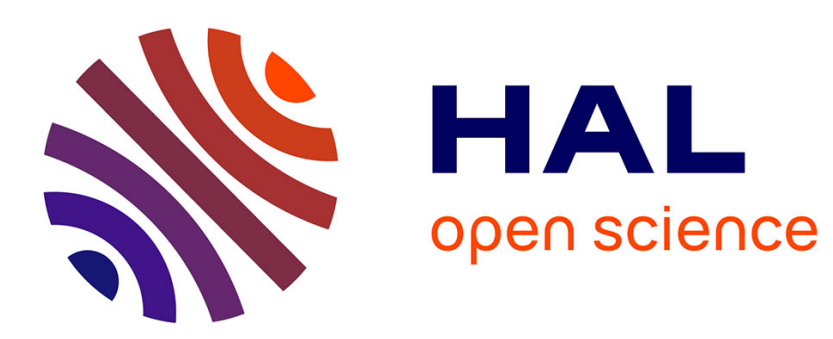

\title{
UNANIMOUS IMPLEMENTATION: A CASE FOR APPROVAL MECHANISMS
}

\author{
Matias Nunez, Dimitrios Xefteris
}

\section{To cite this version:}

Matias Nunez, Dimitrios Xefteris. UNANIMOUS IMPLEMENTATION: A CASE FOR APPROVAL MECHANISMS . 2016. hal-01270275v2

\section{HAL Id: hal-01270275 \\ https://hal.science/hal-01270275v2}

Preprint submitted on 30 Jun 2016

HAL is a multi-disciplinary open access archive for the deposit and dissemination of scientific research documents, whether they are published or not. The documents may come from teaching and research institutions in France or abroad, or from public or private research centers.
L'archive ouverte pluridisciplinaire HAL, est destinée au dépôt et à la diffusion de documents scientifiques de niveau recherche, publiés ou non, émanant des établissements d'enseignement et de recherche français ou étrangers, des laboratoires publics ou privés. 


\title{
UNANIMOUS IMPLEMENTATION: A CASE FOR APPROVAL MECHANISMS*
}

\author{
MATÍAS NÚÑEZ ${ }^{\mathrm{a}}$ AND DIMITRIOS XEFTERIS ${ }^{\mathrm{b}}$
}

\begin{abstract}
Aвstract. We design the class of (indirect) Approval mechanisms which allow the players' strategies to coincide with the subsets of the outcome space. By focusing on the single-peaked domain, we prove that: a) Each of these mechanisms is characterized by a unique equilibrium outcome, and b) for almost every implementable welfare optimum (outcome of a social choice rule), including the Condorcet winner alternative, there exists an Approval mechanism that unanimously implements it. That is, Approval mechanisms help a society achieve every feasible welfare goal, and, perhaps more importantly, they promote social coherence: The implemented outcome is approved by everyone.
\end{abstract}

KeYwords. Nash Implementation, Strategy-proof, Unanimity, Indirect Mechanisms.

Jel Classification. C9, D71, D78, H41.

\section{INTRODUCTION}

Democratic entities, once they set their fundamental welfare goals, try to achieve them by adopting decision-making procedures that allow for the equal participation of all individuals. These democratic decision-making procedures may be broadly split into two categories: voting and deliberation. Voting requires agents to take actions in support of certain policy alternatives. Given the type of actions and the voting rule at play, an alternative is implemented. In the literature, a voting mechanism is a - simultaneous or sequential - game with a formal structure, whose unique equilibrium outcome ${ }^{1}$ coincides with a specific welfare optimum. That is, the voting mechanism selects the outcome of a social choice rule (Maskin [1999]). Indeed, in standard decision-making frameworks well-defined voting mechanisms exist and result in a variety of welfare optima and outcomes of social choice rules. For example, in the context of single-peaked preferences, as recently shown by Gershkov et al. [2015], sequential quota mechanisms may implement any - generalized - median rule.2. On the other hand, deliberation requires agents to engage in rounds of informal discussions

\footnotetext{
${ }^{a}$ CNRS \& Université Paris Dauphine, France.

b University of Cyprus, Cyprus.

Date: June 30, 2016.

* We thank Larbi Alaoui, Elchanan Ben Porath, Anna Bogomolnaia, Geoffroy de Clippel, Marie-Pierre Dargniès, Gabrielle Demange, Edith Elkind, Marc Fleurbaey, Thibault Gajdos, Jean-François Laslier, Mark Le Quement, Antonin Macé, Debasis Mishra, Eduardo Perez-Richet, Carlos Pimienta, Clemens Puppe, Marion Oury, John Quah, Ludovic Renou, Alessandro Riboni, Ariel Rubinstein, Remzi Sanver, Alejandro Saporiti, Thomas Sjöström, Vasiliki Skreta and Olivier Tercieux for useful discussions and conference and seminar participants for their comments. We are particularly indebted to Françoise Forges and Yves Sprumont for the detailed comments on the paper. Matías acknowledges financial support from the LABEX MME-DII (ANR11-LBX-0023-01) and from the ANR-14-CE24-0007-01 CoCoRICo-CoDec.

${ }^{1}$ If, for certain individual preferences, we care to implement a certain welfare optimum, then we cannot rely on procedures that admit other equilibrium outcomes: Voting games that admit multiple equilibrium outcomes are uninteresting from a Nash implementation perspective (see Maskin [1999]).

${ }^{2}$ The welfare optimum defined by such a rule coincides with the median of the set which consists of: a) the voters' ideal policies and b) some exogenously fixed numbers (phantom voters).
} 
and negotiations until a consensual decision is reached. These procedures guarantee that the outcome reflects the interests of all members (Innes and Booher [1999]), and they are employed in high-stakes decision-making by institutions such as the UN Security Council and the European Council.

Both of these approaches to democracy have deep philosophical grounds, and, despite apparent differences, they complement each other. Arriving at unanimous decisions through deliberations is usually deemed superior to voting since it leads to collective harmony, but it is also more costly. As a result, a collective decision problem is commonly solved by voting when the costs of deliberation surpass the potential costs of post-decision confrontation and clash. When a collective body is composed of a small number of entities (such as the countries of the EU) among which clash and confrontation who have been historically costly (for example, the two world wars), it is straightforward that deliberation is the optimal choice..$^{3}$ When a collective body, though, is too large for deliberation to take place in an effective manner and/or a costly clash among the participating members is not unlikely, voting mechanisms are more likely to be adopted.

Since the advantage of deliberative democracy is the implementation of a consensual alternative and the minimization of the likelihood that the participating entities will clash following the decision (at the expense of reaching this decision after a possibly long period), while the advantage of voting is the low cost of decision-making (at the expense possibly generating post-decision conflicts), would it not be desirable to implement welfare optima via voting mechanisms that generate unanimous outcomes?

In this paper, we focus on the framework of single-peaked preferences, $4^{4}$ and we design the class of (indirect) Approval mechanisms which do precisely this: They bring together the described positive features of voting (low decision-making costs) and deliberation (unanimous decisions). These mechanisms allow every player to support as many alternatives as one wants (an arbitrary interval within the unit interval). After all individuals report their sets of approved alternatives, a publicly known aggregation rule is applied, and an alternative is implemented. These aggregation rules might take very simple forms. The most intuitive examples are arguably the median and the mean aggregation rule. When players submit their sets of approved alternatives a distribution of approvals is generated: The density of this distribution at $x \in[0,1]$ is identical to the number of individuals that has approved of alternative $x$, normalized by the total measure of approvals. The median (resp. mean) aggregation rule simply implements the median (resp. mean) of this distribution.

Our main finding is that, under some mild restrictions, every implementable welfare optimum may be unanimously implemented by some anonymous Approval mechanism. An Approval

\footnotetext{
${ }^{3}$ Moreover, elected officials that take decisions using advice from committees of experts are much more comfortable following unanimous recommendations than suggestions that are disputed by a number of experts in the committee. Unanimous recommendations minimize the responsibility of the decision maker and make him less accountable to groups of citizens that are negatively affected by his decisions. In addition, when experts agree on a policy recommendation, it is hard for elected officials to succumb to interest groups' pressures and neglect experts' advice, and this should maximize the probability of informed decision-making. These are only a few additional reasons that the consensus building literature was developed (see, for example, Bessette [1980], Gutmann and Thompson [1996], Gutmann and Thompson [2002] and Fishkin and Laslett [2003]). ${ }^{4}$ The set of alternatives is $A=[0,1]$, and the set of possible preference relations consists of the single-peaked ones in $A$.
} 
mechanism is understood to unanimously implement a welfare optimum/social choice rule if: a) it implements it in every Nash equilibrium, and b) there is at least one equilibrium in which each player includes in his strategy (set of approved outcomes) the implemented outcome. The equilibrium strategies of most players take an easy "I approve every alternative at most (least) as large as the implemented alternative" form. In fact, every player with a preferred alternative to the left (right) of the implemented one approves the implemented alternative and all the alternatives to its left (right). That is, in equilibrium, at most one player will not include the implemented outcome and his own ideal outcome in his strategy, and this player's ideal outcome must coincide with the implemented one. Hence, every equilibrium is substantially unanimous in the sense that, for each voter, the implemented outcome and his ideal one either are both contained in his strategy or they coincide with each other.

Notice that in the context of single-peaked voting, the implementable welfare optima essentially coincide with the outcomes of (generalized) median rules. Indeed, as proved by Moulin [1980] (generalized) median rules are the unique social choice rules that satisfy efficiency and strategy-proofness, while Berga and Moreno [2009] established that strategyproof rules which are "not too bizarre" (in the context of Sprumont [1995]) $)^{5}$ are the only implementable ones. This allows us to provide a transparent characterization of the unique equilibrium outcome of each Approval mechanism. ${ }^{6}$ Moreover, it gives us the tools to design explicitly an Approval mechanism for each (generalized) median rule - including one for the pure median rule (also known as the Condorcet rule or, simply, majority rule). Hence, we explain how to construct an Approval mechanism that implements any given (generalized) median rule, and we provide the Approval mechanism that unanimously implements the ideal policy of the median voter (Condorcet winner alternative). To our knowledge, this is the first simple simultaneous game that implements the Condorcet winner alternative and arguably is of interest on its own. Finally, the fact that in equilibrium, players approve, not only the implemented outcome, but their ideal one as well, indicates that these rules, beyond unanimity, promote sincere revelation of preferences.

The Approval mechanisms can be applied to a variety of decision-making problems. Consider for example a number of judges who disagree on the quality of an athletic performance (say, in gymnastics or in figure skating) and who have to jointly assign a score to this performance, while each of them wants the joint score to be as close as possible to his individual performance evaluation. Another potential, and - perhaps - more important application, is the determination of London Interbank Offered Rate (LIBOR) or the board members of the European Central Bank (ECB) deciding over the interest rate from a closed and convex set of interest rates (see Cai [2009], Rausser et al. [2015] and Rosar [2015] among others for

\footnotetext{
${ }^{5}$ That is, we restrict attention to anonymous rules that implement each of the alternatives for at least one full-range preference profile.

${ }^{6}$ Our context is a public good provision one. In the context of private good provision, Bochet et al. [2008] perform a related exercise with the uniform rule as characterized by Sprumont [1991] and Ching [1994] More precisely, they prove for a large class of allocation rules, at each preference profile, there is a unique Nash equilibrium allocation that coincides with the uniform allocation for the profile.
} 
recent analysis)..$^{7}$ Our Approval mechanisms can be of interest in these settings since they can improve the quality of decision-making by ensuring a unanimous final decision.

In what follows we discuss the relevant literature (section 2), we describe the model (section 3) and present an example (section 4). Then we formally analyze the unanimous implementation through approval mechanisms (section 5).

\section{Relevant Literature}

We aim to show the usefulness of indirect mechanisms in encouraging unanimous agreements. To do so, we focus on the single-peaked domain and prove the following result: Using approval mechanisms, we can unanimously implement any anonymous, efficient and strategy-proof social choice function. The remainder of this section reviews the related literature and underlines our contribution to the implementation theory.

Recall that, as proved by Moulin [1980], a peak-only social choice function is efficient, anonymous and strategy-proof if and only if it is a generalized median rule (GMR) with $(n-1)$ phantoms. ${ }^{8}$ This is among the few general positive results in social choice theory. Its interpretation is not very intuitive since the meaning of the phantoms or fixed ballots is at first sight far from clear. To clarify, we will now briefly explain how these rules work. We assume throughout that the outcome space $A$ is the interval $[0,1]$. A GMR $f$ is characterized by the phantom vector $\left(p_{1}, \ldots, p_{n-1}\right)$ : Given the peaks $\left(t_{1}, \ldots, t_{n}\right)$ of the voters, it selects $f\left(t_{1}, t_{2}, \ldots, t_{n}\right)$ as an outcome with

$$
f\left(t_{1}, t_{2}, \ldots, t_{n}\right)=m\left(t_{1}, t_{2}, \ldots, t_{n}, p_{1}, p_{2}, \ldots, p_{n-1}\right),
$$

such that each phantom $p_{i}$ is in the interval $[0,1]$ and $m(\cdot)$ stands for the median. $\left.\cdot 9\right]$ Note that each phantom is not required to be in the interior of $A$. That plays a key role. Indeed, if $p_{i}=0$ for any $i=1, \ldots, n-1$, then

$$
f\left(t_{1}, t_{2}, \ldots, t_{n}\right)=\min \left(t_{1}, t_{2}, \ldots, t_{n}\right),
$$

\footnotetext{
${ }_{7}$ The London Interbank Offered Rate (LIBOR) is the interest rate at which banks can borrow from each other and plays a critical role in financial markets. LIBOR anchors contracts amount "to the equivalent of $\$ 45000$ for every human being on the planet" (see MacKenzie [2008]). The banks are asked to submit an interest rate at which their banks could borrow money. The lowest and highest quarter of the values are discarded and the Libor corresponds to the average of the remainder. In other words, the device used to determine this index is the trimmed mean rule. Theorists have mostly focused on the pure mean rule (without trimming) and their conclusion over its properties is qualified (see Renault and Trannoy [2005] and Yamamura and Kawasaki [2013] for theoretical works on this subject and Marchese and Montefiori|[2011] and Block et al. [2014] for experimental ones). In environments with a large number of voters, this rule seems to be a natural candidate as it is the unique one satisfying a weakening of strategy-proofness (see Ehlers et al. [2004]). While the latter feature is quite desirable, the former violates the usual desiderata of voting theory: A voter drops his most preferred policy to announce an extremist policy that maximizes his impact on the final outcome. This extreme polarization of the voters' positions seems to posit a fundamental problem with the average method.

${ }^{8}$ One may wonder how restrictive the peak-only assumption is. Arguably, a lot of information is neglected by restricting attention from the preference relations to just their peaks. However, this intuition turns out to be false: When preferences are single-peaked, it turns out that every strategy-proof rule with an interval range must be peak-only. See Ching [1997] and Sprumont [1995] for a direct proof and Barberà et al. [1993] for an extension in a multidimensional discrete model.

${ }^{9}$ Moulin's original work assumes that the outcome space is the set of real numbers. Our results can be extended to such a framework.
} 
whereas if $p_{i}=1$ for any $i=1, \ldots, n-1$, then

$$
f\left(t_{1}, t_{2}, \ldots, t_{n}\right)=\max \left(t_{1}, t_{2}, \ldots, t_{n}\right) .
$$

More interestingly, when $n$ is odd, letting $p_{i}=0$ for any $i \leq \frac{n-1}{2}$ and $p_{i}=1$ for any $i \geq \frac{n-1}{2}+1$ leads to

$$
f\left(t_{1}, t_{2}, \ldots, t_{n}\right)=m\left(t_{1}, t_{2}, \ldots, t_{n}\right),
$$

and, hence, coincides with the Condorcet winner alternative (pure median rule). A similar reasoning shows that - carefully selecting the phantoms - leads to the implementation of the $k^{\text {th }}$ ranked type.

We consider generic GMRs, the ones in which interior phantoms are all non-identical. That is, a GMR is non-generic if there are some $p_{i}, p_{j} \in(0,1)$ with $p_{i}=p_{j}$. The class of generic $G M R$ s is of particular interest since we prove that the approval mechanisms unanimously implement these rules.

While these rules are obviously anonymous, it is less evident that they are also efficient and strategy-proof. Efficiency arises from having not more than $n-1$ phantoms and, hence, ensuring that the final outcome lies in the interval defined by the lowest and the highest type. Strategy-proofness holds since no agent strictly prefers to misreport his type independently of the announcements of the rest of the players. Indeed, if a voter's peak is to the left of the chosen alternative, any announcement different than his peak has two possible consequences: either it makes the final decision greater than the chosen alternative or it does not affect the decision (see Border and Jordan [1983] regarding the notion of uncopromisingness). This naturally implies that the game generated by each $G M R$ has a very appealing Nash equilibrium in which every voter sincerely reveals his true peak.

However, one should note that the game generated by each GMR need not lead to the generalized median of the true peaks. In this respect, the GMRs share a common feature with other strategy-proof mechanisms: They admit a large multiplicity of Nash equilibria, some of which produce different outcomes. For instance, the game triggered by the pure median rule exhibits a large set of equilibria: As long as every player announces the same alternative $x$, this constitutes an equilibrium that implements $x$ since no unilateral deviation affects the aggregate outcome 10 This leads to the following conclusion: The direct game associated to a GMR does not Nash implement the GMR (see Repullo [1985] for similar results).

When presented with the previous observation, two main questions appear: (i) Why would we care about implementing GMRs? and (ii) how should we implement them?

As far as the first question is concerned, a careful examination of the literature shows that these rules are essentially the only Nash implementable ones in this environment. To see why, consider the following line of reasoning. First, Maskin [1999] proves that any Nash implementable social choice function must be Maskin monotonic (for any domain of preferences). Second, Berga and Moreno [2009] prove that with single-peaked preferences,

\footnotetext{
${ }^{10}$ Experimental evidence shows that strategy-proof mechanisms need not lead a large share of the agents to reveal their true type (see Attiyeh et al. [2000], Kawagoe and Mori [2001], Kagel and Levin [1993] and Cason et al. [2006] among others).
} 
a rule is Maskin monotonic if and only if it is strategy-proof and (weakly) non-bossy. ${ }^{11}$ Moreover, they prove that non-bossiness is equivalent to convex range in this preference domain (note that the GMRs have convex range). If $f$ is implementable but $f$ is not a GMR, then it is strategy-proof rule without a convex range. If a rule has a non-convex range, then it fails unanimity in the sense that an alternative need not be implemented even if it is the most preferred one of all voters. This constitutes a strong argument against the use of rules without a convex range. As Sprumont [1995] puts it, the GMRs are the only implementable rules which are not "too bizarre" in this environment. In other words, if the social planner's objective is to implement an efficient, unanimous and anonymous social choice function, he must opt to implement a GMR.

In order to answer the second question, the natural answer would be the use of the integer game. In this game, the players send messages to the social planner; to ensure efficiency, the players name integers. When their messages contradict each other, the one announcing the largest integer is rewarded. Yet, the integer games were built to be applicable in very general settings rather than for their plausibility. For this reason, these mechanisms are often quite complex, and this has motivated researchers to investigate the implementation problem using different approaches, as argued by Jackson [2001].

The literature on designing appealing indirect mechanisms is vast. Yet, it often lacks general results, while succeeding in tailoring interesting mechanisms for particular situations. In this literature, the most closely related contributions are the ones by Yamamura and Kawasaki [2013] and Gershkov et al. [2015] and the one by Saijo et al. [2007].

Yamamura and Kawasaki [2013] proves how to implement GMRs through a class of simple direct mechanisms: the average rules. As they show, the agents tend to adopt an extremist behavior (either 0 or 1 ) in equilibrium. Moreover, the equilibrium outcome coincides with the GMR of the true peaks with an important restriction: All phantoms must be interior (i.e. different from 0 or 1). Hence, one cannot implement the Condorcet winner using the average rules. Gershkov et al. [2015] show how to implement the GMRs through sequential quota mechanisms. More precisely, their sequential mechanisms are obtained by modifying a sequential voting scheme suggested by Bowen [1943]. Our approach is orthogonal to theirs since our Approval mechanisms are simultaneous. In short, the implementation results that these papers obtain are related to ours: Yet, our main contribution is to show that Approval mechanisms give incentives for reaching unanimous agreements.

Once we have commented on these closely related works, we will state some final remarks on two literatures to which this paper is connected.

The first one is the one focusing on strategic voting and, more precisely, on the unanimity rule (see Feddersen and Pesendorfer [1996, 1997, 1998] for classical references in the area and Koriyama and Szentes [2009] and Bouton et al. [2016] for recent contributions). The comparison between the current results and the ones in such a literature seems to be

\footnotetext{
${ }^{11}$ The relation between strategy-proofness and Maskin monotonicity has produced a rich literature. Muller and Satterthwaite [1977] show that Maskin monotonicity and strategy-proofness are equivalent when preferences are the unrestricted. Dasgupta et al. [1979] obtains strategy-proofness as necessary condition for Maskin monotonicity under some restricted preferences. For recent contributions, see also Takamiya [2007] and Klaus and Bochet [2013].
} 
far from pertinent. Indeed, in broad terms, these works often evaluate the consequences of honest and strategic behavior of voters when confronted with the unanimity rule. They tend to perform a welfare analysis of this rule in several settings: private/common values, complete/incomplete information, optimal size of the jury, etc.. Their main message is that the unanimity rule tends to be inefficient whenever strategic voting is present: That is, voters do not reveal their true information if the collective decision is to be made by unanimity. Our objective is different: We posit incentives to endogenously achieve unanimity assuming from the outset that voters are strategic. Having said so, our paper is also related to the literature on Approval Voting (see Brams and Fishburn [1983], Laslier and Sanver [2010] and Bouton and Castanheira 2012] among others), to which Approval mechanisms borrow both its name and its flexibility. Laslier et al. [2015] design a bargaining device over lotteries based on Approval voting and derive conditions for consensus reaching in equilibrium with just two agents.

Finally, this work is, of course, related to implementation theory (see Maskin [1999] and Jackson [2001] for a review). Our notion of implementation is related to the one of Nash implementation in the sense that it requires that all equilibria of the game form implement the desired social choice rule ${ }^{12}$ It is stronger than Nash implementation since it requires the existence of unanimous equilibrium in which all voters agree on the implemented policy. Our objective is hence two-fold: to reduce the multiplicity of equilibria associated to strategy-proof mechanisms while ensuring a unanimous agreement. The former requirement is closely related to the contribution by Saijo et al. [2007] who proposed a novel concept of implementation named secure implementation. This implementation notion ${ }^{13}$ aims to get rid of the multiplicity of equilibria inherent to the direct mechanisms associated with strategy-proof rules previously described by coarsening the notion of implementation. Their proposal manages to derive securely implementable functions in some situations (such as quasi-linear preferences) but fails to do so in our framework. Our contribution is hinting at a possible manner of overcoming this theoretical objection: In order to implement a strategy-proof social choice function, rather than using its associated direct mechanism one could make use of indirect Approval mechanisms that foster unanimity while ensuring the uniqueness of the equilibrium outcome.

\section{The SETting}

Let $A:=[0,1]$ denote the set of alternatives, and $N:=\{1, \ldots, n\}$ with $n \geq 2$ stand for the finite set of players. Each player is endowed with preferences over $A$. The utility for player $i$ when $x \in A$ is the implemented policy equals $u_{i}(x)$ with $u_{i}: A \rightarrow \mathbb{R}$, where each $u_{i} \in U$,

\footnotetext{
${ }^{12}$ There is a large literature on implementation, and different notions have been proposed. While Nash implementation is arguably the most well-known, scholars have focused on other concepts, such, Bayesian implementation (Jackson [1991]), virtual implementation (Abreu and Sen [1991], Bergemann and Morris [2009b]), implementation in mixed strategies (Mezzetti and Renou| [2012]) and implementation with partial honesty (Dutta and Sen [2012]) among others.

${ }^{13}$ More specifically, they focus on a dual notion of implementation that requires dominant strategy implementation and Nash implementation simultaneously. The notion of secure implementation is equivalent to the one of robust implementation (see Bergemann and Morris [2009a] among others) in any private values setting, as ours, as shown by Adachi [2014].
} 
the set of single-peaked preferences. Note that each player $i$ has a unique peak denoted $t_{i}$ so that $u_{i}\left(x^{\prime}\right)<u_{i}\left(x^{\prime \prime}\right)$ when $x^{\prime}<x^{\prime \prime} \leq t_{i}$ and when $t_{i} \leq x^{\prime \prime}<x^{\prime}$.14 We let $\left(t_{1}, \ldots, t_{n}\right)$ stand for a distribution of the players' peaks, and $u=\left(u_{1}, \ldots, u_{n}\right) \in \mathscr{U}:=\prod_{j=1}^{n} U$.

A social choice function is a function $f: \mathscr{U} \rightarrow A$ that associates every $u \in \mathscr{U}$ with a unique alternative $f(u)$ in $A$. A mechanism is a function $\theta: S \rightarrow A$ that assigns to every $s \in S$, a unique element $\theta(s)$ in $A$, where $S:=\prod_{i=1}^{n} S_{i}$ and $S_{i}$ is the strategy space of agent $i$. The mechanism $\theta$ is the direct revelation mechanism associated to a SCF $f$ if $S_{i}=U_{i}$ for all $i \in N$ and $\theta(u)=f(u)$ for every $u \in U$. A SCF $f$ is strategy-proof if for all $i \in N$, all $u_{i}, \tilde{u}_{i} \in U_{i}$, and all $u_{-i} \in U_{-i}, u_{i}\left(f\left(u_{i}, u_{-i}\right)\right) \geq u_{i}\left(f\left(\tilde{u}_{i}, u_{-i}\right)\right)$. As shown by Moulin [1980], these rules admit a simple characterization: The median of the peaks of the players plus $(n-1)$ exogenous parameters (phantoms). More formally, for any finite collection of points $x_{1}, \ldots, x_{m}$ in $[0,1]$, we let $m\left(x_{1}, \ldots, x_{m}\right)$ denote their median. That is, the smallest number $m\left(x_{1}, \ldots, x_{m}\right) \in x_{1}, \ldots, x_{m}$, which satisfies: $\frac{1}{m} \#\left\{x_{i} \mid x_{i} \leq m\left(x_{1}, \ldots, x_{m}\right)\right\} \geq \frac{1}{2}$ and $\frac{1}{m} \#\left\{x_{i} \mid x_{i} \geq m\left(x_{1}, \ldots, x_{m}\right)\right\} \geq \frac{1}{2}$. In the domain $\mathscr{U}$ and assuming that each agent's message is one element of $A$, a $S C F f$ is anonymous, efficient and strategy-proof if and only if there exist $(n-1)$ real numbers, $\kappa_{1}, \ldots, \kappa_{n-1}$ such that $f\left(t_{1}, \ldots, t_{n}\right)=m\left(t_{1}, \ldots, t_{n}, \kappa_{1}, \ldots, \kappa_{n-1}\right)$.

We let $\mathscr{B}$ denote the collection of closed intervals of $A$ and define an approval mechanism as a mechanism such that $S_{i}=\mathscr{B}$ for every $i \in N \sqrt{15}$ We write $\underline{b_{i}}=\min b_{i}$ and $\overline{b_{i}}=\max b_{i}$ for each $b_{i} \in \mathscr{B}$. Note that the strategy set $\mathscr{B}$ allows elements of different dimensions: singletons and positive length intervals. To accommodate this fact, we let $\lambda_{d}$ denote the Lebesgue measure on $\mathbb{R}^{d}$ with $d=0,1$. Since each $b_{i}$ is a convex set, its dimension is well-defined so that for each approval profile $b=\left(b_{i}, b_{-i}\right)$, we let $\operatorname{dim}(b)=\max _{i \in N} \operatorname{dim}\left(b_{i}\right)$.

Given a mechanism $\theta: S \rightarrow A$, the strategy profile $s \in S$ is a Nash equilibrium of $\theta$ at $u \in \mathscr{U}$, if $u_{i}\left(\theta\left(s_{i}, s_{-i}\right)\right) \geq u_{i}\left(\theta\left(s_{i}^{\prime}, s_{-i}\right)\right.$ for all $i \in N$ and any $s_{i}^{\prime} \in S_{i}$. Let $N^{\theta}(u)$ be the set of Nash equilibria of $\theta$ at $u$. The mechanism $\theta$ implements the $S C F f$ in Nash equilibria if for each $u \in \mathscr{U}$, (i) there exists $s \in N^{\theta}(u)$ such that $\theta(s)=f(u)$ and (ii) for any $s \in N^{\theta}(u)$, $\theta(s)=f(u)$. The SCF $f$ is implementable if there exists a mechanism that implements $f$ in Nash equilibria. An Approval Mechanism $\theta$ unanimously implements the SCF $f$ if (i) $\theta$ implements $f$ in Nash equilibria and (ii) there exists $b \in N^{\theta}(u)$ such that $\cap_{i=1}^{n} b_{i}=\{x\}$ and with $\theta(b)=x$ for some $x \in A$. Our focus is on the unanimous implementation of strategyproof rules.

\section{An Example: the Median Approval Mechanism}

In order to clarify the main ideas behind unanimous implementation, this section presents an example that illustrates how an approval mechanism works. While our results can be generalized, we restrict ourselves to three voters in this section to clarify the logic underlying our more general approach.

\footnotetext{
${ }^{14}$ For simplicity, we assume that $t_{i} \neq t_{j}$ for any $i, j \in N$. Our results are not affected when relaxing this constraint.

${ }^{15}$ This assumption can be relaxed by allowing any finite union of closed and convex subsets of $A$ to be the set of pure strategies. Relaxing it however would imply more cumbersome notation and proofs since then two strategies that differ by a zero-measure set can have equivalent consequences. Moreover, it does not affect greatly the result. We prefer to stick to the simpler definition of strategy.
} 
Let $N=\{1,2,3\}$ and $0<t_{1}<t_{2}<t_{3}<1$ denote their respective ideal points. 16 The Approval mechanisms that we consider throughout have the following common structure: a) Every player simultaneously and independently announces a closed interval $b_{i}$ in $A, \mathrm{~b}$ ) these intervals define a score distribution, and c) the mechanism implements $\theta(b)$ with $b=\left(b_{1}, \ldots, b_{n}\right)$ which equals some quantile of the score distribution such as the median.

The Approval mechanisms differ in how this score distribution is generated. While the general structure is discussed in the rest of the paper, we stick here to the simplest interesting manner: That is, we assume that when voter $i$ submits the interval $b_{i}$, he assigns an individual score $s_{x}\left(b_{i}\right)$ to each $x \in[0,1]$ as follows:

$$
s_{x}\left(b_{i}\right)=1 \text { for any } x \in b_{i} \text { and } s_{x}\left(b_{i}\right)=0 \text { otherwise. }
$$

Collectively, each profile $b$ assigns a score $s_{x}(b)$ to each alternative $x$ which equals the sum of the individual scores so that $s_{x}(b)=\sum_{i=1}^{n} s_{x}\left(b_{i}\right)$. If at least one player submits a positive interval, the score distribution is the function $\phi: \mathscr{B}^{n} \times[0,1] \rightarrow[0,1]$ such that

$$
\phi(b, z)=\int_{0}^{z} \frac{s_{x}(b)}{\sum_{i=1}^{n}\left(\overline{b_{i}}-\underline{b_{i}}\right)} d x .
$$

The Median Approval mechanism associates any profile $b$ with the median $\theta(b)$ of the score distribution (when $\phi$ is continuous, $\phi(b, \theta(b))=\frac{1}{2}$, while when all voters announce a singleton, $\theta(b)$ corresponds to the median of these singletons).

To understand why this mechanism triggers unanimous agreements, it is key to see that the best responses are particularly simple to define. Indeed, take some profile $b=\left(b_{i}, b_{-i}\right)$ and assume that $b_{i}$ is a best response for player $i$. If $t_{i}<\theta(b)$, recall that the agent's best response(s) is the strategy that minimizes $\theta(b)$ since preferences are single-peaked. In this case, his unique best response is $b_{i}=[0, \theta(b)]$. Indeed, it is intuitive that any best response for player $i$ must include some points to the left of $\theta(b)$. Now, assume that some best response $b_{i}^{\prime}$ satisfies $b_{i}^{\prime} \cap[\theta(b), 1] \neq \emptyset$. Then consider the strategy $c_{i}$ such that $c_{i}=b_{i} \cap[0, \theta(b)]$. It follows that $\theta\left(c_{i}, b_{-i}\right)<\theta\left(b_{i}^{\prime}, b_{-i}\right)$ since the only difference between $c_{i}$ and $b_{i}^{\prime}$ is that $c_{i}$ does not include any point to the right of $\theta(b)$, proving that $b_{i}^{\prime}$ cannot be a best response. It follows that any best response for player $i$ must be included in $[0, \theta(b)]$. Take now some $b_{i}^{\prime}$ with $b_{i}^{\prime} \neq[0, \theta(b)]$. Then, again this cannot be a best response since $\theta(b)<\theta\left(b_{i}^{\prime}, b_{-i}\right)$ since $b_{i} \backslash b_{i}^{\prime}$ consists only of alternatives located to the left of $\theta(b)$. Therefore, if $t_{i}<\theta(b)$, the unique best response for player $i$ is to play $[0, \theta(b)]$. By a symmetric argument, when $t_{i}>\theta(b)$, the unique best response is to play $[\theta(b), 1]$. These best responses fully describe the strategic behavior in any equilibrium in which no player obtains his peak as the implemented outcome. They are the main culprit for the unanimous implementation since a rational player must include the outcome in his ballot.

In order to illustrate the previous best responses, let $N=\{1,2,3\}$ and $t_{1}, t_{2}<\frac{1}{3}<t_{2}$. The game triggered by this mechanism admits a unique equilibrium $b^{*}$ with $b_{1}^{*}=b_{2}^{*}=\left[0, \frac{1}{3}\right]$ and

\footnotetext{
${ }^{16} \mathrm{~A}$ similar example is analyzed in Austen-Smith and Banks 2005, chapter 6, p.233. In their model, the three players also reach a consensus over an interior policy in the interval $[0,1]$. The reasons for consensus depend on the discount factors, which define the no-delay equilibrium. See Banks and Duggan [2000] for a bargaining model of collective choice.
} 
$b_{3}^{*}=\left[\frac{1}{3}, 1\right]$ and $\theta\left(b^{*}\right)=\frac{1}{3}, 17$ Note that in $b^{*}$, every player is playing his unique best response since he approves of all the alternatives to the left or to the right of the implemented outcome. Figure 1 depicts the distribution of approvals generated by $b^{*}$. The alternatives lower than $\frac{1}{3}$ are selected by two players whereas the rest of them just by one. Hence it is simple to understand that $\theta\left(b^{*}\right)=\frac{1}{3}$ since it splits the area below the score function in two exact halves.

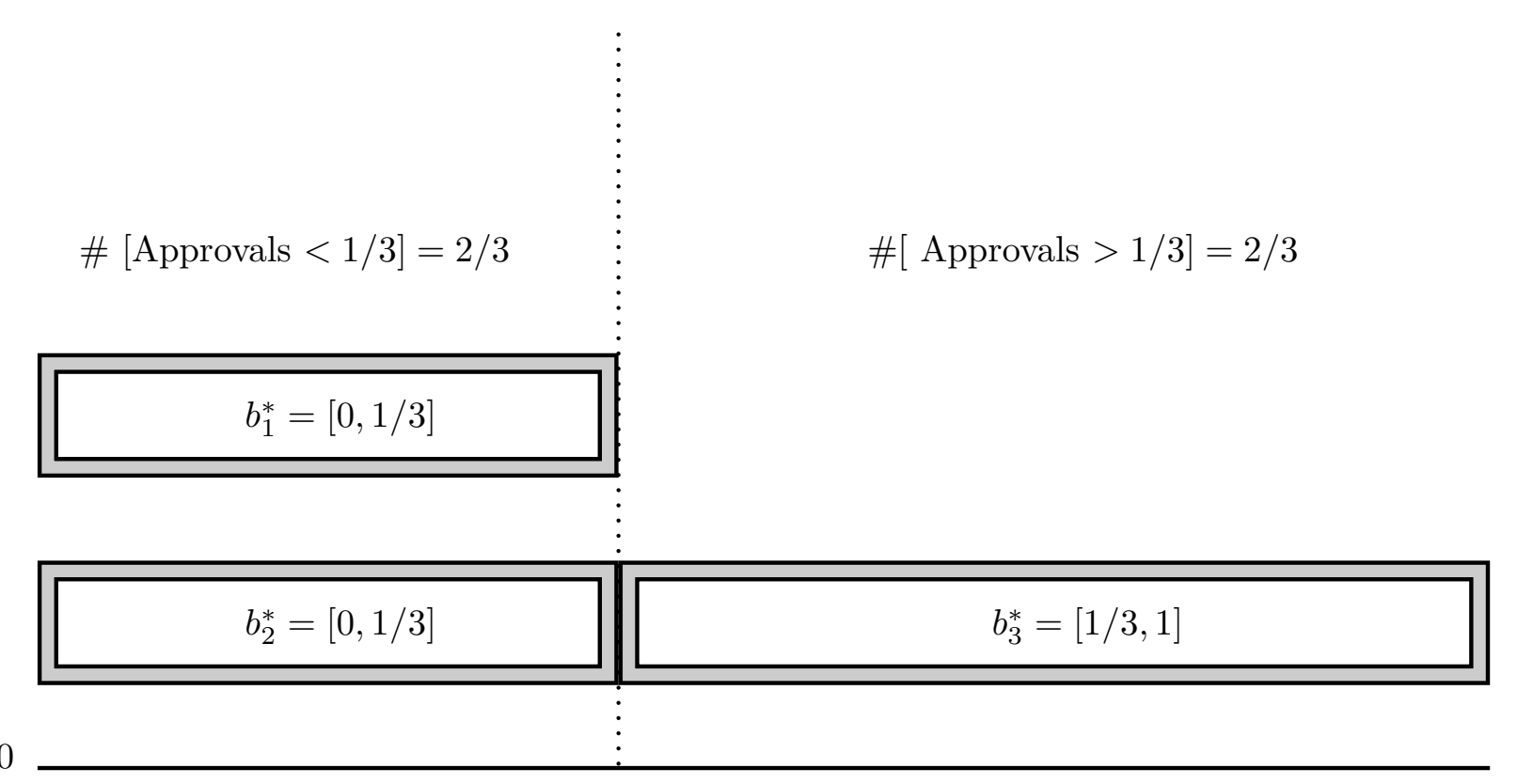

$$
1 / 3=\theta\left(b^{\prime}\right)
$$

Figure 1. Distribution of approvals associated to $b^{*}$.

Consider the most intuitive deviations $b_{1}^{\prime}$ for player 1 : either $[0, x]$ with $x>1 / 3$ or $[0, y]$ with $y<1 / 3$. The first one can be labeled as overshooting since the player includes alternatives that exceed the outcome $\frac{1}{3}$ whereas the second one is undershooting. Letting $b^{\prime}=\left(b_{1}^{\prime}, b_{2}^{*}, b_{3}^{*}\right)$, Figure 2 shows that both of these deviations move the outcome to the right of $\frac{1}{3}$ and hence are not best responses. A similar intuition applies to the rest of the deviations.

As depicted by Figure $2 \mathrm{a}$, overshooting moves the outcome to the right of $\frac{1}{3}$ since the area to the left of $\frac{1}{3}$ is equal to $\frac{\frac{2}{3}}{1+x}$ which is smaller than $\frac{1}{2}$ for any $x>\frac{1}{3}$. This is not a best response for player 1 since his peak is located to the left of $\frac{1}{3}$. Indeed, overshooting expands the total area while leaving unchanged the area to the left of $\frac{1}{3}$. It is then simple to understand that the median must move to the right of $\frac{1}{3}$.

A similar argument applies to undershooting and is depicted by Figure $2 \mathrm{~b}$. Again, the area to the left of $\frac{1}{3}$ is smaller than $\frac{1}{2}\left(\frac{1}{3}+y<\frac{2}{3}\right)$ so that undershooting also moves the outcome to the right of $\frac{1}{3}$. When undershooting, the player's deviation reduces both the total area and the area to the left of $\frac{1}{3}$ by the same amount making impossible that the median is located to the left of $\frac{1}{3}$. A similar argument shows that the unique best response for player 1 is to approve of $\left[0, \frac{1}{3}\right]$ : no under- or over-shooting occurs in equilibrium.

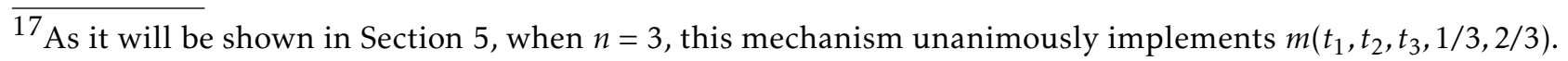




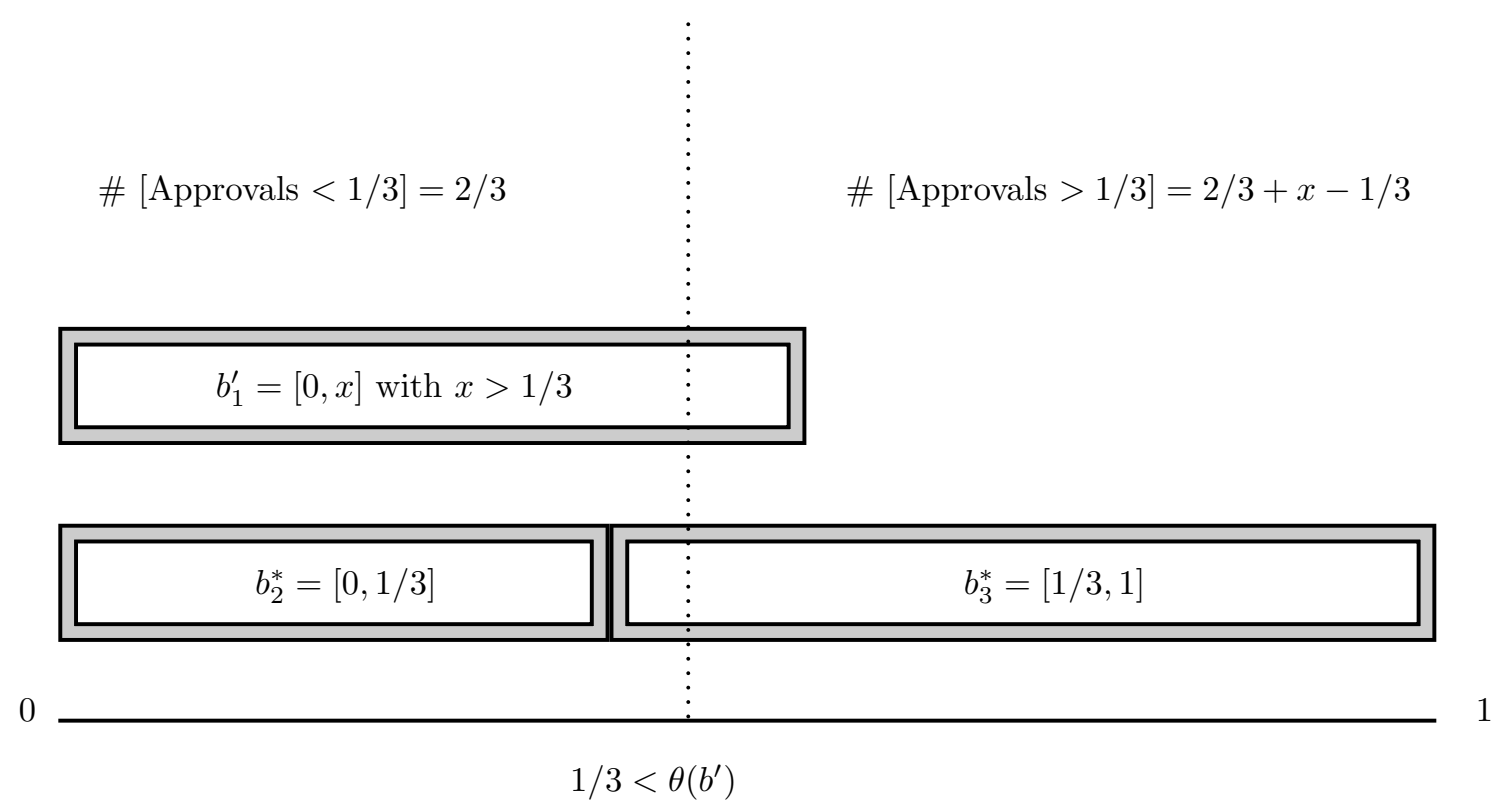

(A) Over-shooting in $b^{*}$.

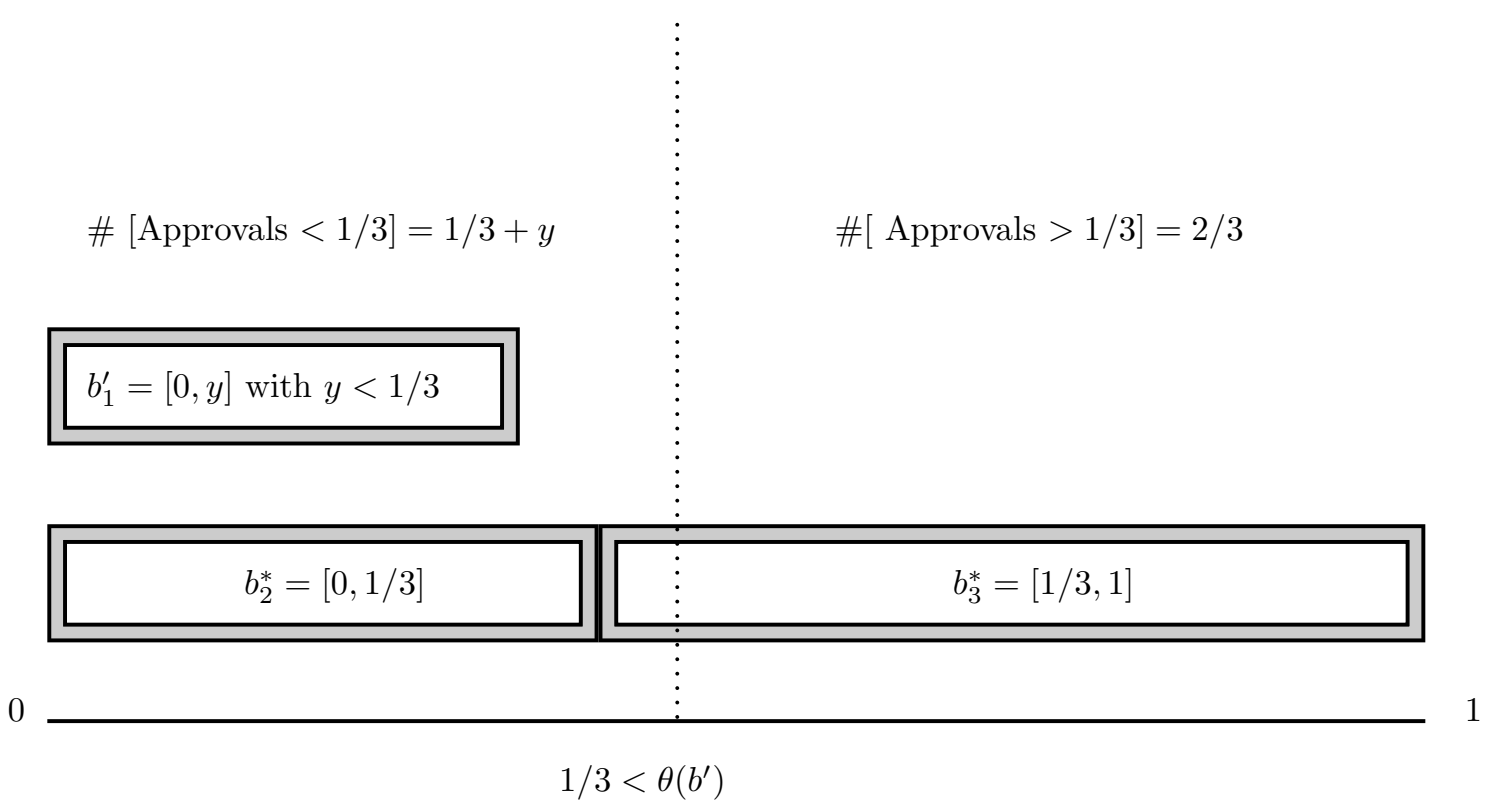

(в) Under-shooting in $b^{*}$.

Figure 2. Deviations for Player 1 in $b^{*}$

\section{UNANimOUS IMPLEMENTATION}

This section presents the main results of this work. First, we describe certain properties of Approval mechanisms that are sufficient for unanimous implementation. Then, we prove by construction (as in Maskin [1999]) that for each generic GMR, an Approval mechanism that unanimously implements it exists.

5.1. Properties of Approval Mechanisms . We restrict ourselves to anonymous Approval mechanisms ${ }^{18}$ such that for each $x \in A$, there is some $b \in \mathscr{B}^{n}$ with $\theta(b)=x$. It is important to stress here that our characterization approach is not a normative one (we do not try to single

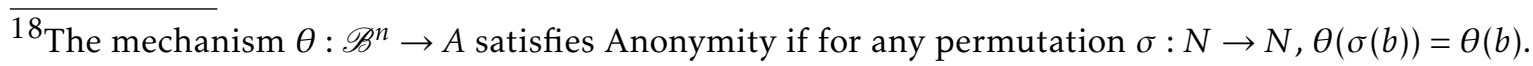


out a set of mechanisms that satisfy certain appealing properties) but a consequentialistic one (we try to pin down properties of Approval mechanisms that are adequate for unanimous outcomes). Indeed, this kind of a characterization, by its very nature, cannot precede the precise identification of mechanisms that lead to the desired outcomes and, hence, the current presentation order might appear, at first, as a hysteron proteron. One could claim, though, that the actual order in which results are derived and the optimal order in which these results are presented, do not necessarily coincide. Starting with a thorough description of the features of Approval mechanisms that are behind unanimity is, to ours' view, more enlightening and insightful, than a direct presentation of the relevant mechanisms and a subsequent attempt to detail the underlying incentive structure.

The first property deals with the two sort of strategies allowed in an Approval mechanism. Indeed, either a strategy contains finitely many alternatives (zero-dimensional strategy) or infinitely many (one-dimensional strategy). One might argue that zero-dimensional strategies are stubborn in the sense that the player is approving of a zero-measure set of the set of available alternatives. Similarly, a one-dimensional strategy is a compromise in opposition to stubborn strategies. The set of stubborn and compromise strategies are respectively labeled by $S(\mathscr{B})$ and $C(\mathscr{B})$ with $\mathscr{B}=S(\mathscr{B}) \cup C(\mathscr{B})$. The Approval Mechanisms on which we will focus give incentives to players to select one-dimensional strategies, in the following sense:

Incentives for Compromise (IC): The mechanism $\theta: \mathscr{B}^{n} \rightarrow A$ satisfies Incentives for Compromise if, for any $i \in N$ and for any $b_{i} \in S(\mathscr{B}), \exists b_{i}^{\prime} \in C(\mathscr{B})$ with $u_{i}\left(\theta\left(b_{i}^{\prime}, b_{-i}\right)\right)>u_{i}(\theta(b))$ whenever $\theta(b) \neq t_{i}$.

This property ensures that a player has an incentive to submit a compromise strategy rather than a stubborn one, as long as the mechanism does not select his most preferred alternative. The main implication of $I C$ is that there is no equilibrium in which each player announces a singleton.

In order to define our second property, we introduce the following piece of notation. For each $i \in N$ and any $b_{-i} \in \mathscr{B}^{n-1}, \theta\left(\mathscr{B}, b_{-i}\right)$ denotes the attainable set of player $i$ at $b_{-i}$; It represents the set of available alternatives that player $i$ can choose when the rest of the players select $b_{-i}$. Since $\mathscr{B}$ is not finite, the set $\theta\left(\mathscr{B}, b_{-i}\right)$ need not have a maximum or a minimum. Monotonicity gives precise conditions to characterize the maximum and the minimum of the attainable set when they exist.

Monotonicity $(M O N):$ The mechanism $\theta: \mathscr{B}^{n} \rightarrow A$ satisfies Monotonicity if for any $i \in N$ and any $b_{-i} \in \mathscr{B}^{n-1}$, we have:

$$
\begin{gathered}
b_{i}^{m} \in \arg \min \theta\left(\mathscr{B}, b_{-i}\right) \text { if and only if } b_{i}^{m}=\left[0, x_{i}^{m}\right] \text { with } x_{i}^{m}=\theta\left(\left[0, x^{m}\right], b_{-i}\right), \text { and } \\
b_{i}^{M} \in \arg \max \theta\left(\mathscr{B}, b_{-i}\right) \text { if and only if } b_{i}^{M}=\left[x_{i}^{M}, 1\right] \text { with } x_{i}^{M}=\theta\left(\left[x^{M}, 1\right], b_{-i}\right) .
\end{gathered}
$$

That is, when a player attempts to draw an implemented outcome as far left as possible, he should not approve of outcomes to its right and it should not be the case that he does not approve of outcomes to its left, and vice versa. 
For any profile $b \in \mathscr{B}^{n}$, we let $\operatorname{Supp}(b)=\bigcup_{i=1}^{n} b_{i}$ denote the support of $b$. The support denotes the set of alternatives that are selected by at least some player. When the support is convex, all alternatives located between the minimum and the maximum of the support are approved by at least one individual.

The next property requires Approval mechanisms to be continuous, at least with reference to strategy profiles with convex support.

Continuity (C). The mechanism $\theta: \mathscr{B}^{n} \rightarrow A$ satisfies Continuity if for any $i \in N$, any $b \in$ $C(B)^{n}$ and any $b^{\varepsilon}=\left(b_{i}^{\varepsilon}, b_{-i}\right) \in C(B)^{n}$,

$$
\operatorname{Supp}(b), \operatorname{Supp}\left(b^{\varepsilon}\right) \text { are convex and } \lim _{\varepsilon \rightarrow \infty} b_{i}^{\varepsilon}=b_{i} \Longrightarrow \lim _{m \rightarrow \infty} \theta\left(b^{\varepsilon}\right)=\theta(b) .
$$

The continuity property states that the Approval mechanism is continuous with respect to each component as long as the support is convex. This implies that all alternatives between the minimum and the maximum of the support are approved by at least one individual; in equilibrium, the support is convex, whereas when all voters announce singletons, the support is not convex.

To define our final property, we turn attention to the following class of strategy profiles.

For any $j=0,1, \ldots, n$ and any $x \in[0,1]$, we let $\mathscr{B}(j, x):=\left\{b \in \mathscr{B}^{n} \mid \#\left\{b_{i}=[0, x]\right\}=n-j\right.$ and $\left.\left.\#\left\{b_{i}=[x, 1]\right\}=j\right\}\right\}$ denote the set of profiles in which $n-j$ players choose the strategy $[0, x]$ and $j$ players use the strategy $[x, 1]$. Since $\theta$ is anonymous, note that $\theta(b)=\theta\left(b^{\prime}\right)$ for any $b, b^{\prime} \in \mathscr{B}(j, x)$. We now let for any $j \in\{0, \ldots, n\}, K_{j}:=\{x \in A \mid \theta(b)=x$ for any $b \in \mathscr{B}(j, x)\}$.

We are now ready to define the phantoms of an Approval mechanism.

Definition 1. For any $b \in \mathscr{B}(j, x)$ and any Approval mechanism $\theta$, the vector of phantoms $\kappa=$ $\left(\kappa_{j}\right)_{i=0}^{n}$ is defined as follows:

$$
\kappa_{j}:= \begin{cases}0 & \text { if } K_{j}=\varnothing \text { and } \theta(b)<x \forall x \in(0,1), \\ 1 & \text { if } K_{j}=\varnothing \text { and } \theta(b)>x \forall x \in(0,1), \\ K_{j} & \text { if } K_{j} \neq \varnothing \text { and } K_{j} \cap\{0,1\}=\varnothing, \\ \varnothing \quad \text { otherwise. }\end{cases}
$$

Whenever $\kappa_{j}$ is treated like a number, it is implicitly assumed that it is a singleton. We consider that $\kappa_{j}$ is: a) interior if $\kappa_{j} \in(0,1)$ and b) intersective if $\theta(b)$ crosses the 45 degree line at $\kappa_{j} \in(0,1)$ for any $b \in \mathscr{B}(j, x)$. For simplicity, we say that $\kappa_{j}$ denotes the fixed points of $\theta(b)$ for any $b \in \mathscr{B}(j, x)$.

Fixed-Point Monotonicity (FP). The mechanism $\theta: \mathscr{B}^{n} \rightarrow A$ satisfies Fixed-Point Monotonicity if a) all $\kappa_{j}$ s are singletons and increase in $j \in\{0,1, \ldots, n\}$ with $\kappa_{0}=0$ and $\kappa_{n}=1$, and b) every interior $\kappa_{j}$ is distinct and intersective.

This property establishes that the fixed points of the Approval mechanisms are wellbehaved and is essential to ensure the existence of a pure strategy equilibrium. 
5.2. Sufficiency. Equipped with the previous results we are now ready to state the sufficient conditions for unanimous implementation.

Theorem 1. If an Approval Mechanism $\theta$ satisfies $C, F P, M O N$ and $I C$, then:

(1) there is an equilibrium in pure strategies for every admissible preference profile;

(2) in every equilibrium $b$ of $\theta$ we have $\theta(b)=m\left(t_{1}, t_{2}, \ldots, t_{n}, \kappa_{1}, \ldots, \kappa_{n-1}\right)$; and

(3) there is an equilibrium $b$ of $\theta$ with $\cap_{i=1}^{n} b_{i}=\theta(b)$.

Proof. Take some $\theta: \mathscr{B}^{n} \rightarrow[0,1]$ satisfying $C, F P, M O N$ and $I C$. For short, we write $(t, \kappa)$ rather than $\left(t_{1}, t_{2}, \ldots, t_{n}, \kappa_{1}, \ldots, \kappa_{n-1}\right)$. The proof first states the existence of equilibrium (Step A.), then the uniqueness of the equilibrium outcome (Step B.) and finally the existence of a unanimous equilibrium (Step C.).

Step A.: There is some equilibrium $b$ of $\theta$ with $\theta(b)=m(t, \kappa)$.

Step A. is divided into two cases: There is either no $t_{h}$ with $t_{h}=m(t, \kappa)$ (Step A.I.), or there is a $t_{h}$ like we develop in Step A.II.

Step A.I. $\nexists: t_{h}$ with $t_{h}=m(t, \kappa)$. Since there is no $t_{h}$ with $t_{h}=m(t, \kappa)$, there must exist $j \in\{1, \ldots, n-1\}$ such that $\kappa_{j}=m(t, \kappa)$. Therefore, the number of elements located below and above $\kappa_{j}$ in $(t, \kappa)$ is equal to $n-1$, which is equivalent to:

$$
\underbrace{\#\left\{i \in N \mid t_{i}<\kappa_{j}\right\}+(j-1)}_{\text {elements lower than } \kappa_{j}}=\underbrace{\#\left\{i \in N \mid t_{i}>\kappa_{j}\right\}+(n-j-1)}_{\text {elements higher than } \kappa_{j}}=n-1 .
$$

The previous equalities jointly imply that $\#\left\{i \in N \mid t_{i}<\kappa_{j}\right\}=n-1$ and $\#\left\{i \in N \mid t_{i}>\kappa_{j}\right\}=j$. Let $b \in \mathscr{B}^{n}$ be an approval profile with:

$$
b_{i}:= \begin{cases}{\left[0, \kappa_{j}\right]} & \text { if } t_{i}<\kappa_{j}, \\ {\left[\kappa_{j}, 1\right]} & \text { if } t_{i}>\kappa_{j} .\end{cases}
$$

Since $\#\left\{i \in N \mid t_{i}>\kappa_{j}\right\}=j$, it follows that $\#\left\{i \in N \mid t_{i}<\kappa_{j}\right\}=n-j$. Therefore, $b \in \mathscr{B}\left(j, \kappa_{j}\right)$. Moreover, $\theta(b)=\kappa_{j}$ due to $F P$, and, that $\theta(b)=m(t, \kappa)$. In order to prove that $b$ is an equilibrium, assume that there is some $i \in N$ with a profitable unilateral deviation $b_{i}^{\prime}$, so that $\theta\left(b_{i}^{\prime}, b_{-i}\right) \neq \theta(b)$. Assume first that $\theta\left(b_{i}^{\prime}, b_{-i}\right)<\theta(b)$. If $t_{i}>\kappa_{j}$ and given that preferences are single-peaked, it follows that $u_{i}\left(\theta\left(b_{i}^{\prime}, b_{-i}\right)\right)<u_{i}\left(\theta\left(b_{i}, b_{-i}\right)\right)$. In other words, $b_{i}^{\prime}$ is not a profitable deviation, entailing a contradiction. If $t_{i}<\kappa_{j}$, then by definition $b_{i}=\left[0, \kappa_{j}\right]$. However, due to $M O N, b_{i}$ is player $i$ 's unique best response, which proves that there is no profitable deviation. The same argument applies if $\theta\left(b_{i}^{\prime}, b_{-i}\right)>\theta(b)$, which proves that $b$ is an equilibrium of the game and concludes Step A.I.

Step A.II. $\exists: t_{h}$ with $t_{h}=m(t, \kappa)$. If there exists $j \in\{1, \ldots, n-1\}$ such that $\kappa_{j}=t_{h}$, then $j=n-h$ or $j=n-h+1$. Using the same line of reasoning as in A.I., one can show that: a) when $j=n-h+1$, any $b \in \mathscr{B}\left(n-h+1, t_{h}\right)$ is an equilibrium with $\theta(b)=t_{h}$ and b) when $j=n-h$, any $b \in \mathscr{B}\left(n-h, t_{h}\right)$ is an equilibrium with $\theta(b)=t_{h}$.

If $t_{h}=m(t, \kappa)$ and $t_{h} \neq \kappa_{j}$, there are $n-1$ values smaller than $t_{h}$ in $(t, \kappa)$. There are essentially two cases here: a) $t_{h} \in\left(\kappa_{1}, \kappa_{n-1}\right)$ and b) $t_{h}<\kappa_{1}$ (the proof for the case $t_{h}>\kappa_{n-1}$ is symmetric). Below, we consider both cases in turn. 
a) Choose $j$, such that $1<j<n-1$, with $\kappa_{j}<t_{h}=m(t, \kappa)<\kappa_{j+1}$. Moreover $\#\left\{\kappa_{l} \mid \kappa_{l}<t_{h}\right\}=j$ and $\#\left\{i \in N \mid t_{i}<t_{h}\right\}=h-1$ so that: $j+h-1=n-1 \Longrightarrow j=n-h$. Therefore, $\kappa_{n-h}<t_{h}<\kappa_{n-h+1}$.

For each $A \in \mathscr{B}$, we define $b^{A}$ as the approval profile with:

$$
b_{i}^{A}= \begin{cases}{\left[0, t_{h}\right]} & \text { if } t_{i}<t_{h} \\ A & \text { if } t_{i}=t_{h} \\ {\left[t_{h}, 1\right]} & \text { if } t_{i}>t_{h} .\end{cases}
$$

Our objective is to prove that there is some $b^{A}$ with $\theta\left(b^{A}\right)=t_{h}$. Since $\theta$ is continuous over a player's strategy, the result immediately follows from the Intermediate Value Theorem provided that there are some $A^{\prime}$ and $A^{\prime \prime}$ with

$$
\theta\left(A^{\prime}, b_{-h}^{A}\right)<t_{h}<\theta\left(A^{\prime \prime}, b_{-h}^{A}\right)
$$

By $C$ and $F P$, we have that for any $b \in \mathscr{B}\left(n-h, \kappa_{n-h}\right), \theta(b)=\kappa_{n-h}<t_{h}$ if $\kappa_{n-h} \in(0,1)$ and for any $b \in \mathscr{B}\left(n-h, t_{h}\right), \theta(b)<t_{h}$ if $\kappa_{n-h}=0$. Similarly, for any $b \in \mathscr{B}\left(n-h+1, \kappa_{n-h+1}\right)$, $\theta(b)=\kappa_{n-h+1}>t_{h}$ if $\kappa_{n-h+1} \in(0,1)$, and for any $b \in \mathscr{B}\left(n-h+1, t_{h}\right), \theta(b)>t_{h}$ if $\kappa_{n-h+1}=1$. Hence, $\theta\left(A^{\prime}, b_{-h}^{A}\right)<t_{h}$ when $A^{\prime}=\left[0, t_{h}\right]$ and $\theta\left(A^{\prime \prime}, b_{-h}^{A}\right)>t_{h}$ when $A^{\prime}=\left[t_{h}, 1\right]$ so that there exists some $A$ with $\theta\left(b^{A}\right)=t_{h}$.

In order to prove that this profile ( $b$ for short) is an equilibrium, suppose by contradiction that there exists some $i \in N$ with a profitable deviation $b_{i}^{\prime}$. Then, it cannot be the player with type $t_{h}$ since $\theta(b)=t_{h}$. Suppose then that $\theta\left(b_{i}^{\prime}, b_{-i}\right)<\theta(b)$. Then, $t_{i}<t_{h}$; otherwise, if $t_{i}>t_{h}$ then $u_{i}\left(b_{j}^{\prime}, b_{-j}\right)<u_{i}\left(b_{i}, b_{-i}\right)$, a contradiction with $b_{i}^{\prime}$ being a profitable deviation. However, any voter with $t_{i}<t_{h}$ is playing his unique best response [0, $\left.t_{h}\right]$, entailing again a contradiction. A symmetric argument applies when $\theta\left(b_{i}^{\prime}, b_{-i}\right)>\theta(b)$. Therefore $b$ must be an equilibrium concluding a) in Step A.

b) In this case, $t_{h}=m(t, \kappa)<\kappa_{1}$, and hence, $h=n$. According to $F P$, we have that for any $b \in \mathscr{B}(n-h, x), \theta(b)<x$ for every $x \in(0,1)$ (because $n-h=0<1)$ and for any $b \in \mathscr{B}(n-h+1, x)$, $\theta(b)(x)=x$ if and only if $x=\kappa_{1}$ (because $\left.n-h+1=1\right)$. Therefore, $\theta\left(\left[0, t_{h}\right], b_{-h}\right)<t_{h}$ and $\theta\left(\left[t_{h}, 1\right], b_{-h}\right)>t_{h}$, and, hence, the continuity arguments guarantee here the existence of an interval $A^{*}$ such that $\theta\left(b^{A^{*}}\right)=t_{h}$. This, in turn, ensures the existence of an equilibrium similar to the one described in a), which concludes the proof of step A.

Step B.: Any equilibrium $b$ of $\theta$ satisfies $\theta(b)=m(t, \kappa)$. Suppose that, there is some $\theta$ that admits an equilibrium $b$ with $\theta(b)>m(t, \kappa)$. We let $L_{m}:=\left\{i \in N \mid t_{i} \leq m(t, \kappa)\right\}$ and $F_{m}:=\{j \in$ $\left.\{1, \ldots, n-1\} \mid \kappa_{j} \leq m(t, \kappa)\right\}$ with $\# L_{m}=i^{\prime}$ and $\# F_{m}=j^{\prime}$. However, by definition, it must be the case that $i^{\prime}+j^{\prime} \geq n$ so that $n-i^{\prime} \leq j^{\prime}$. Thus, $\kappa_{n-i^{\prime}} \leq \kappa_{j^{\prime}}=m(t, \kappa)(i)$. By Monotonicity, the unique best response for any player in $S$ equals $[0, \theta(b)]$ so that $\theta(b) \leq \kappa_{n-i^{\prime}}(i i)$. Combining both (i) and (ii), it follows that $\theta(b) \leq m(t, \kappa)$ a contradiction with $\theta(b)>m(t, \kappa)$. A symmetric claim delivers also a contradiction whenever $\theta(b)<m(t, \kappa)$, proving that $\theta(b)=m(t, \kappa)$ as wanted.

Step C.: There exists some equilibrium $b$ of $\theta$ with $\cap_{i=1}^{n} b_{i}=\theta(b)$. Note that by construction, the equilibrium built in Step A.I satisfies this claim. In Step A.II, $n-1$ players announce $t_{h}$ in their equilibrium strategy. Hence it suffices to show that there is some $A^{*}$ with $t_{h} \in A^{*}$. If player $h$ plays $\left[0, t_{h}\right]$, the outcome is lower than $t_{h}$ whereas if he plays $\left[t_{h}, 1\right]$ then the outcome is higher than $t_{h}$ as proved in Step A.II. Observe that if $h$ plays $[\underline{c}, \bar{c}]$ and we start from 
$\underline{c}=0$ and $\bar{c}=t_{h}$ and first we start increasing $\bar{c}$ from $t_{h}$ to 1 and then $\underline{c}$ from 0 to $t_{h}$, we should have $i$ ) always $t_{h}$ is included in the interval $[\underline{c}, \bar{c}]$ and $\left.i i\right)$ due to the continuity of the outcome in $\underline{c}$ and $\bar{c}$ the outcome should equal to $t_{h}$ for some strategy.

Q.E.D.

As a by-product of the previous Theorem and the continuity property, we can establish the following interesting property of Approval mechanisms: These mechanisms are partially revealing in the sense that any player always has a best response in which he approves of his peak $t_{i}$.

Lemma 1 (Partially Revealing). Let $\theta: \mathscr{B}^{n} \rightarrow$ A satisfy $C, F P, M O N$ and $I C$. For any approval profile $b$ and any $i \in N$, there is some best response $b_{i} \in \mathscr{B}$ with $t_{i} \in b_{i}$.

The proof is an immediate consequence of Monotonicity whenever $\theta(b) \neq t_{i}$. If $\theta(b)=t_{i}$, the claim is a consequence of $\theta$ being a deterministic mechanism as well of, the different properties of the Approval mechanisms.

5.3. Feasibility. We now prove that the properties defined in the necessity part are not vacuous in the sense that for any generic $G M R$, there exists some Approval mechanism that unanimously implements it.

Let $\eta:[0,1] \rightarrow[0,1]$ be a differentiable and strictly increasing function with $\eta(0)=0$ and $\eta(1)=1$ and $q$ a non-negative real number.

We assume that when voter $i$ submits the interval $b_{i}$, he is endowed with up to $q+\left(\eta\left(\overline{b_{i}}\right)-\right.$ $\left.\eta\left(\underline{b_{i}}\right)\right)$ points to be distributed over $b_{i}$.

More precisely, if $\operatorname{dim}\left(b_{i}\right) \geq \operatorname{dim}(b)$, then the strategy $b_{i}$ assigns an individual score $s_{x}\left(b_{i}, q, \eta\right)$ to each $x \in[0,1]$ as follows:

$$
s_{x}\left(b_{i}, q, \eta\right)=\frac{q}{\overline{b_{i}}-\underline{b_{i}}}+\eta^{\prime}(x) \text { for any } x \in b_{i} \text { and } s_{x}\left(b_{i}\right)=0 \text { otherwise, }
$$

so that his total weight $s\left(b_{i}, q, \eta\right)=\int_{0}^{1} s_{x}\left(b_{i}, q, \eta\right) d x$ is equal to $q+\left(\eta\left(\overline{b_{i}}\right)-\eta\left(\underline{b_{i}}\right)\right)$ as defined. On the contrary, when $\operatorname{dim}\left(b_{i}\right)<\operatorname{dim}(b)$, so that $b_{i}$ is a singleton and some other voter announces a positive dimension interval, we let $s_{x}\left(b_{i}, q, \eta\right)=0$ so that his vote is not taken into account.

Collectively, each profile $b$ assigns a score $s_{x}(b, q, \eta)$ to each alternative $x$ which equals the sum of the individual scores so that $s_{x}(b, q, \eta)=\sum_{i=1}^{n} s_{x}\left(b_{i}, q, \eta\right)$.

If all voters announce a singleton, we let $\operatorname{med}\left(b_{1}, b_{2}, \ldots, b_{n}\right)$ be the outcome. On the contrary, if some voter announces a positive-dimension interval, the score distribution is the function $\phi_{q, \eta}: \mathscr{B}^{n} \times[0,1] \rightarrow[0,1]$ such that

$$
\phi_{q}(b, \eta, z)=\int_{0}^{z} \frac{s_{x}(b, q, \eta)}{\sum_{i=1}^{n} s\left(b_{i}, q, \eta\right)} d x .
$$

The Approval mechanism associates any profile $b$ with the alternative $\theta_{\alpha, q, \eta}(b)$ such that

$$
\theta_{\alpha, q, \eta}(b):=\min \left\{x \in[0,1] \mid \phi_{q}\left(b, \eta, \theta_{q, \eta}(b)\right)=\alpha\right\} .
$$

In other words, it selects the $\alpha$-quantile of the sample endogenously generated by $b$ given $q$ and $\eta$. Each mechanism $\theta_{\alpha, q, \eta}$ is characterized by $q, \eta$ and the parameter $\alpha$. It is called a Generalized Approval Mechanism (GAM). 
As shown by the next Lemma, any $\theta_{\alpha, q, \eta}$ is a well-defined mechanism.

Lemma 2. For any approval profile $b=\left(b_{i}, b_{-i}\right)$, any non-negative $q$ and any $\eta:[0,1] \rightarrow[0,1]$, $\theta_{\alpha, q, \eta}(b)$ is a well-defined mechanism.

Proof. Note first that $\frac{s_{x}(b, q, \eta)}{\sum_{i=1}^{n} s\left(b_{i}, q, \eta\right)} \geq 0$ for any $x \in[0,1]$. It suffices to show that its integral over $[0,1]$ equals 1 , which is equivalent to $\phi_{q}(b, \eta, 1)=1$. But this is satisfied since $\int_{0}^{1} s_{x}(b, q, \eta) d x=$ $\sum_{i=1}^{n} s\left(b_{i}, q, \eta\right)$.

Q.E.D.

Proposition 1. Any GAM satisfies IC, MON, C and FP.

While the formal proof of this proposition for $I C, M O N$ and $C$ is relegated to the appendix, we do not prove that FP is satisfied by any GAM. Note that the GAMs are designed to exhibit trackable phantoms so that FP holds by construction.

Indeed, the vector of fixed points of each mechanism $\theta_{\alpha, q, \eta}$ is denoted by $\kappa^{\alpha, q, \eta}$ and is defined as follows. To simplify notation, we write $\kappa$ and $\theta$ and assume that $\alpha, q$ and $\eta$ are given. Recall that for any $j=1, \ldots, n-1$, any strategy profile $b \in \mathscr{B}(j, x)$ has $n-j$ players using the strategy $[0, x]$ and $j$ players using the strategy $[x, 1]$. As in definition 1 , each $\kappa_{j}$ is defined as follows

$$
\kappa_{j}:= \begin{cases}0 & \text { if } K_{j}=\varnothing \text { and } \theta(b)<x \forall x \in(0,1), \\ 1 & \text { if } K_{j}=\varnothing \text { and } \theta(b)>x \forall x \in(0,1), \\ K_{j} & \text { if } K_{j} \neq \varnothing \text { and } K_{j} \cap\{0,1\}=\varnothing, \\ \varnothing & \text { otherwise. }\end{cases}
$$

Therefore, we have for any $j=1, \ldots, n-1$, if $K_{j} \neq \emptyset$ and $K_{j} \cap[0,1]$, then any phantom $\kappa_{j}$ must satisfy the following inequality:

$$
\frac{(n-j) \eta\left(\kappa_{j}\right)+(n-j) q}{q n+(n-j) \eta\left(\kappa_{j}\right)+j\left[1-\eta\left(\kappa_{j}\right)\right]}=\alpha \Leftrightarrow \eta\left(\kappa_{j}\right)=\frac{\alpha(n q+j)-(n-j) q}{(n-j)-\alpha(n-2 j)} .
$$

It follows that the phantom vector $\kappa=\left(\kappa_{j}\right)_{j=0}^{n}$ associated with each GAM satisfies

$$
\kappa_{j}=\max \left\{0, \min \left\{1, \eta^{-1}\left(\frac{\alpha(n q+j)-(n-j) q}{(n-j)-\alpha(n-2 j)}\right)\right\}\right\},
$$

for each $j \in\{1, \ldots, n-1\}$. Note that for any $j$ such that $\eta^{-1}\left(\frac{\alpha(n q+j)-(n-j) q}{(n-j)-\alpha(n-2 j)}\right) \in(0,1)$, then $\kappa_{j}=$ $\eta^{-1}\left(\frac{\alpha(n q+j)-(n-j) q}{(n-j)-\alpha(n-2 j)}\right)$. Since $\eta$ is differentiable and strictly increasing, one can prove that $0 \leq$ $\kappa_{1} \leq \kappa_{2} \leq \ldots \leq \kappa_{n-1} \leq 1$, and that for any pair of interior phantoms $\kappa_{i}, \kappa_{j} \in(0,1)$, it must be the case that $\kappa_{i} \neq \kappa_{j}$. It follows that any GAM satisfies FP.

We are now ready to state the main result of this section.

Theorem 2. For any generic GMR, there exists some GAM that unanimously implements it.

Proof. Take some generic GMR with phantom vector $p=\left(p_{1}, \ldots, p_{n-1}\right)$. We want to prove that there is some $G A M \theta_{\alpha, q, \eta}$ with phantom vector $\kappa$ that unanimously implement the GMR so that $\kappa=p$.

Assume first that that every $p_{j} \in(0,1)$. In this case, it suffices to set $\alpha, q$ and $\eta$ so that: 


$$
p_{j}=\eta^{-1}\left(\frac{\alpha(n q+j)-(n-j) q}{(n-j)-\alpha(n-2 j)}\right)
$$

meaning $\kappa=p$ as wanted.

Assume now that there is some $a, b \in\{1, \ldots, n-1\}$ such that $p_{a}=0$ and $p_{b}=1$ with $p_{i} \in(0,1)$ if $i \in(a, b)$. As previously argued, it must be the case that $p_{1} \leq p_{2} \leq p_{n-1}$. Hence, for any $s \leq a, p_{s}=0$ and for any $t \geq b, p_{t}=1$.

Take now some $q$ and $\alpha$ such that

$$
\frac{\alpha(n q+a)-(n-a) q}{(n-a)-\alpha(n-2 a)}=0 \text { and } \frac{\alpha(n q+b)-(n-b) q}{(n-b)-\alpha(n-2 b)}=1 .
$$

This ensures that $\kappa_{a}=0$ and $\kappa_{b}=1$. The previous equalities are equivalent to

$$
q=\frac{a \alpha}{n(1-\alpha)-a}
$$

where $\alpha$ depends on the value of $a+b$. More precisely, if $a+b=n$ then $\alpha=1 / 2$ whereas,

$$
\alpha=\frac{1}{(n-a-b) n}((n-a)(n-b)-\sqrt{a b(n-a)(n-b)}) \text { if } a+b<n
$$

and

$$
\alpha=\frac{1}{(n-a-b) n}((n-a)(n-b)+\sqrt{a b(n-a)(n-b)}) \text { if } a+b>n
$$

which proves that the equalities (3) are compatible. Moreover, since $0 \leq \kappa_{1} \leq \kappa_{2} \leq \ldots \leq$ $\kappa_{n-1} \leq 1$, it follows that, for any $s \leq a, \kappa_{s}=0$ and for any $t \geq b, \kappa_{t}=1$.

If $b=a+1$, then we are done, since $\kappa=p$. If $b>a+1$, then by assumption, any $p_{i}$ with $i \in(a, b)$ must satisfy $p_{i} \in(0,1)$. Then, given that $q$ and $\alpha$ are given by $(3)$, it is enough to suitably select $\eta$ such that for any $i \in(a, b)$,

$$
p_{i}=\eta^{-1}\left(\frac{\alpha(n q+i)-(n-j) q}{(n-i)-\alpha(n-2 i)}\right)
$$

which ensures that $\kappa=p$ as wanted.

Q.E.D.

5.4. Examples. While the previous arguments remain general, we have not explicitly stated which generic GMRs are unanimously implemented by which GAM. To do so, we now introduce some examples that show the usefulness of the methods proposed throughout. The first one is concerned with the implementation of the Condorcet winner. The second and third prove how to implement GMRs with interior phantoms. While the second one is a $G A M$, the third one is not a GAM: This proves that there might be other interesting classes of Approval mechanisms.

Example 1: Implementing the Condorcet winner. Let $N=\{1,2,3\}$ be the set of voters with $t_{1}<t_{2}<t_{3}$ and set $q=1$ and $\alpha=1 / 2$. Namely, each voter is endowed $1+\overline{b_{i}}-\underline{b_{i}}$ points and the outcome selected corresponds to the median of the distribution generated by $b$. The unique equilibrium outcome of this game is the selection of $t_{2}$, the median of the types and the Condorcet winner policy. 
We first prove how to elect $t_{2}$ at equilibrium and then show why this is the unique equilibrium outcome. To build an equilibrium supporting $t_{2}$, note that the analysis of the best responses imply that, in any such equilibrium, $t_{1}$ plays $\left[0, t_{2}\right]$ whereas $t_{3}$ plays $\left[t_{2}, 1\right]$. We need to prove that there is some $b_{2}^{\prime}$ that ensures $t_{2}$ to be elected. To see why there must exist such a $b_{2}$, let $b^{\prime}=\left(\left[0, t_{2}\right], b_{2}^{\prime},\left[t_{2}, 1\right]\right)$. Then for any $t_{2} \in(0,1)$, if $b_{2}^{\prime}=\left[0, t_{2}\right]$, $\phi_{1}\left(b^{\prime}, t_{2}\right)=\int_{0}^{t_{2}} \frac{2+\frac{2}{t_{2}}}{3+2 t_{2}+1-t_{2}} d x>\frac{1}{2}$, whereas if $b_{2}^{\prime}=\left[t_{2}, 1\right], \phi_{1}\left(b^{\prime}, t_{2}\right)=\int_{0}^{t_{2}} \frac{1+\frac{1}{t_{2}}}{3+t_{2}+2\left(1-t_{2}\right)} d x<\frac{1}{2}$. Therefore, the $t_{2}$-voter can change his $b_{2}^{\prime}$ from $\left[0, t_{2}\right]$ to $\left[t_{2}, 1\right]$ to find a strategy $\left[t_{2}-\varepsilon, t_{2}+\delta\right]$ that contains $t_{2}$ and which leads to $\phi_{1}\left(b, t_{2}\right)=1 / 2$, or, to the unanimous implementation of her ideal policy. For instance, if $t_{2}=\frac{1}{3}, \varepsilon=\frac{7}{30}$ and $\delta=\frac{1}{10}$ ensures this whereas when $t_{2}=\frac{2}{3}$, $\varepsilon=\frac{1}{66}$ and $\delta=\frac{1}{6}$ triggers an outcome equal to $t_{2}$.

Now, in order to prove that there is no other possible equilibrium outcome, assume by contradiction that there is some $x \neq t_{2}$ elected at equilibrium $b$. Assume that $x<t_{2}$, the case with $x>t_{2}$ being symmetric. If $x \in\left(t_{1}, t_{2}\right)$, then the description of the best responses imply that $b=([0, x],[x, 1],[x, 1])$ since $t_{1}<x<t_{2}<t_{3}$. But then $\phi_{1}(b, x)=\int_{0}^{x} \frac{1+\frac{1}{x}}{4+x} d x$ so that $\phi_{1}(b, x)<$ $\frac{1}{2}$ for any $x \in\left(t_{1}, t_{2}\right)$, so that there is no such an equilibrium. If $x \in\left[0, t_{1}\right)$, then $b$ must be such that the tree players play $[x, 1]$ so that the median of these three intervals must be higher than $x$. Finally, if $x=t_{1}$, then in any equilibrium $b$, players 2 and 3 play $b_{2}=b_{3}=\left[t_{1}, 1\right]$. However, if player 1 plays $b_{1}=\left[0, t_{1}\right], \theta_{1}(b)$ then $\theta_{1}\left(\left[0, t_{1}\right],\left[t_{1}, 1\right],\left[t_{1}, 1\right]\right)=\frac{\left(t_{1}+1\right)\left(t_{1}-3\right)}{4\left(t_{1}-2\right)}>t_{1}$ for any $t_{1} \in\left[0, \frac{1}{2}\right]$. Yet, since $\left[0, t_{1}\right]$ would be the best response of any player with ideal policy to the left of $t_{1}$, it follows that $\left[0, t_{1}\right] \equiv \arg \min _{b_{1} \in \mathscr{B}} \theta_{1}\left(b_{1},\left[t_{1}, 1\right],\left[t_{1}, 1\right]\right)$, which implies that $\theta_{1}\left(b_{1},\left[t_{1}, 1\right],\left[t_{1}, 1\right]\right)>t_{1}$ for any $b_{1} \in \mathscr{B}$. Therefore, there is not an equilibrium that elects $t_{1}$. All in all, the unique equilibrium outcome associated with $\theta_{1}$ and $\alpha=\frac{1}{2}$ is $t_{2}$.

Example 2: Interior phantoms with a GAM. If we set $q=0, \alpha=\frac{1}{2}$ and $\eta(x)=x$ for any $x \in[0,1]$. As previously argued, the phantoms of this Approval mechanism must satisfy for any $j=1,2, \ldots, n-1, \eta\left(\kappa_{j}^{q, \eta}\right)=\frac{j+q(2 j-n)}{n} \Longleftrightarrow \kappa_{j}=\frac{j}{n}$. Hence, $N=\{1,2,3\}$ leads to $\kappa_{1}=\frac{1}{3}$ and $\kappa_{2}=\frac{2}{3}$. The equilibria with this mechanism in the case in which $m\left(t_{1}, t_{2}, t_{3}, \frac{1}{3}, \frac{2}{3}\right)$ equals one of the peaks is similar to the ones depicted with the Approval mechanism that implements the Condorcet winner.

However, in the precise case in which $m\left(t_{1}, t_{2}, t_{3}, \frac{1}{3}, \frac{2}{3}\right)=\frac{1}{3}$, the logic is different. Indeed, the mechanism admits a unique equilibrium $b^{*}$ with $b_{1}^{*}=b_{2}^{*}=\left[0, \frac{1}{3}\right]$ and $b_{3}^{*}=\left[\frac{1}{3}, 1\right]$, the one discussed in section 4 . In general, if the equilibrium outcome coincides with a phantom and not with a type, there is a unique equilibrium (all players playing either to the left or to the right of the outcome) whereas this is not the case when a player's peak is the equilibrium outcome (this player can play in several ways, while the rest of the players play either to the left or to the right of the outcome).

Example 3: Interior phantoms without a GAM. For any profile $b$ and any alternative $x$, we let $\#\left\{i \in N \mid x \in b_{i}\right\}$. denote the number of individuals approving of alternative $x$ in $b$.

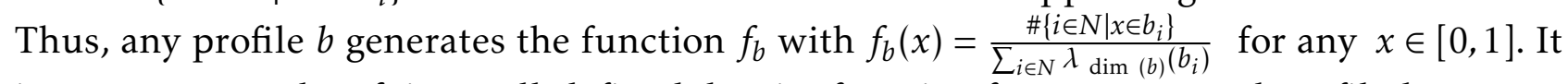
is easy to prove that $f_{b}$ is a well-defined density function for any approval profile $b$. 
We let $\mu_{b}$ stand for the mean of the approval profile $b$ with $\mu_{b}=\int_{[0,1]} x f_{b}(x) d x$. Note that $\mu_{b} \in[0,1]$, and, hence, it always coincides with an alternative. The Average Approval Mechanism associates $\mu_{b}$ to each approval profile $b$ so that $\theta(b)=\mu_{b}$.

One can show that this rule unanimously implements the generic $G M R$ with for any $j=$ $1, \ldots, n-1, \kappa_{j}=\frac{\sqrt{j}}{\sqrt{n-j}+\sqrt{j}}$. This proves that the class of GAMs is not the unique one that implements GMRs.

\section{REFERENCES}

D. Abreu and A. Sen. Virtual implementation in nash equilibrium. Econometrica, 59(4): 997-1021, 1991. 7

T. Adachi. Robust and secure implementation: equivalence theorems. Games and Economic Behavior, 86:96-101, 2014. 7

G. Attiyeh, R. Franciosi, and R. M. Isaac. Experiments with the pivot process for providing public goods. Public choice, 102(1-2):93-112, 2000. 5

D. Austen-Smith and J. Banks. Positive political theory II: strategy and structure, volume 2. University of Michigan Press, 2005. 9

J. Banks and J. Duggan. A bargaining model of collective choice. American Political Science Review, 94(1):73-88, 2000. 9

S. Barberà, F. Gul, and E. Stacchetti. Generalized median voter schemes and committees. Journal of Economic Theory, 61(2):262-289, 1993. 4

D. Berga and B. Moreno. Strategic requirements with indifference: single-peaked versus single-plateaued preferences. Social Choice and Welfare, 32(2):275-298, 2009. 3, 5

D. Bergemann and S. Morris. Robust implementation in direct mechanisms. The Review of Economic Studies, 76(4):1175-1204, 2009a. 7

D. Bergemann and S. Morris. Robust virtual implementation. Theoretical Economics, 4:4588, 2009b. 7

J. Bessette. Deliberative Democracy: The Majority Principle in Republican Government. How Democratic is the Constitution? Washington, D.C., AEI Press., 1980. 2

V. Block, K. Nehring, and C. Puppe. Nash equilibrium and manipulation in a mean rule experiment. Doctoral Dissertation, Karlsruhe Institute of Technology, 2014. 4

O. Bochet, T. Sakai, and W. Thomson. Preference manipulations lead to the uniform rule. Available at SSRN 1220394, 2008. 3

K. C. Border and J. S. Jordan. Straightforward elections, unanimity and phantom voters. The Review of Economic Studies, 50(1):153-170, 1983. 5

L. Bouton and M. Castanheira. One Person, Many Votes: Divided Majority and Information Aggregation. Econometrica, 80:43-87, 2012. 7

L. Bouton, A. Llorente-Saguer, and F. Malherbe. Get rid of unanimity: The superiority of majority rules with veto power. Journal of Political Economy, 2016. forthcoming. 6

H. R. Bowen. The interpretation of voting in the allocation of economic resources. The Quarterly Journal of Economics, pages 27-48, 1943. 6

S.J. Brams and P.C. Fishburn. Approval Voting. Birkhauser, Boston, 1983. 7 
H. Cai. Costly Participation and Heterogeneous Preferences in Informational Committees. The RAND Journal of Economics, 40:173-189, 2009. 3

T.N. Cason, T. Saijo, T. Sjöström, and T. Yamato. Secure implementation experiments: Do strategy-proof mechanisms really work? Games and Economic Behavior, 57:206-235, 2006. 5

S. Ching. An alternative characterization of the uniform rule. Social Choice and Welfare, 11 (2):131-136, 1994. 3

S. Ching. Strategy-proofness and median voters. International Journal of Game Theory, 26(4): 473-490, 1997. 4

P.S. Dasgupta, P.J. Hammond, and E.S. Maskin. The implementation of social choice rules: Some general results on incentive compatibility. Review of Economic Studies, 46:185-216, 1979. 6

B. Dutta and A. Sen. Nash Implementation with Partially Honest Individuals. Games and Economic Behavior, 74:154-169, 2012. 7

L. Ehlers, H. Peters, and T. Storcken. Threshold strategy-proofness: on manipulability in large voting problems. Games and Economic Behavior, 49:103-116, 2004. 4

T. Feddersen and W. Pesendorfer. The Swing Voter's Curse. American Economic Review, 86: 408-424, 1996. 6

T. Feddersen and W. Pesendorfer. Voting Behavior and Information Aggregation in Elections with Private Information. Econometrica, 65:1029-1058, 1997. 6

T. Feddersen and W. Pesendorfer. Convicting the Innocent: The Inferiority of Unanimous Jury Verdicts under Strategic Voting. American Political Science Review, 92:22-35, 1998. 6

J. Fishkin and P. Laslett. Debating Deliberative Democracy. Wiley-Blackwell., 2003. 2

A. Gershkov, B. Moldovanu, and X. Shi. Optimal voting rules. mimeo, University of Bonn, 2015. 1, 6

A. Gutmann and D. Thompson. Democracy and Disagreement. Princeton University Press., 1996. 2

A. Gutmann and D. Thompson. Why Deliberative Democracy? Princeton University Press., 2002. 2

J. E. Innes and D. E. Booher. Consensus building and complex adaptive systems: A framework for evaluating collaborative planning. Journal of the American planning association, pages $412-423,1999$. 2

M. Jackson. Virtual implementation. Econometrica, 59:461-477, 1991.7

M. Jackson. A Crash Course in Implementation Theory. Social Choice and Welfare,, 18:655708, 2001. 6, 7

J. H. Kagel and D. Levin. Independent private value auctions: Bidder behaviour in first-, second-and third-price auctions with varying numbers of bidders. The Economic Journal, 103(419):868-879, 1993. 5

T. Kawagoe and T. Mori. Can the pivotal mechanism induce truth-telling? an experimental study. Public Choice, 108(3-4):331-354, 2001. 5

B. Klaus and O. Bochet. The relation between monotonicity and strategy-proofness. Social Choice and Welfare, 40(1):41-63, 2013. 6 
Y. Koriyama and B. Szentes. A resurrection of the condorcet jury theorem. Theoretical Economics, 4:227-252, 2009. 6

J-F. Laslier, M. Núñez, and C. Pimienta. Reaching Consensus through Simultaneous Bargaining. mimeo, University of New South Wales, 2015. 7

J.F. Laslier and R. Sanver. Handbook on Approval Voting. Heildelberg: Springer-Verlag, 2010. 7

D. MacKenzie. What's in a number? London Review of Books, 2008. URL http: / /www. lrb. co.uk/v30/n18/donald-mackenzie/whats-in-a-number, 4

C. Marchese and M. Montefiori. Strategy versus sincerity in mean voting. Journal of Economic Psychology, 32:93-102, 2011. 4

E. Maskin. Nash Equilibrium and Welfare Optimality. Review of Economic Studies, 66:23-38, 1999. 1, 5, 7, 11

C. Mezzetti and L. Renou. Implementation in mixed nash equilibrium. Journal of Economic Theory, 147(6):2357-2375, 2012. 7

H. Moulin. On Strategy-proofness and Single Peakedness. Public Choice, 35:437-455, 1980. 3, 4,8

E. Muller and M. A. Satterthwaite. The equivalence of strong positive association and strategy-proofness. Journal of Economic Theory, 14(2):412-418, 1977. 6

G. C. Rausser, L. K. Simon, and J. Zhao. Rational Exaggeration and Counter-exaggeration in Information Aggregation Games. Economic Theory, 59(1):109-146, 2015. 3

R. Renault and A. Trannoy. Protecting Minorities through the Average Rule. Journal of Public Economic Theory, 7:169-199, 2005. 4

R. Repullo. Implementation in dominant strategies under complete and in-complete information. Review of Economics Studies, 52:223-229, 1985. 5

F. Rosar. Continuous decisions by a committee: median versus average mechanisms. Journal of Economic Theory, 159- Part A:15-65, 2015. 3

T. Saijo, T. Sjöström, and T. Yamato. Secure implementation. Theoretical Economics, 2:203229, 2007. 6, 7

Y. Sprumont. The division problem with single-peaked preferences: a characterization of the uniform allocation rule. Econometrica: Journal of the Econometric Society, pages 509$519,1991.3$

Y. Sprumont. Strategyproof collective choice in economic and political environments. Canadian Journal of Economics, pages 68-107, 1995. 3, 4, 6

K. Takamiya. Domains of social choice functions on which coalition strategy-proofness and maskin monotonicity are equivalent. Economics Letters, 95(3):348-354, 2007. 6

H. Yamamura and R. Kawasaki. Generalized Average Rules as stable Nash mechanisms to implement generalized median rules. Social Choice and Welfare, 40:815-832, 2013. 4, 6

Appendix A. The class of GAMs

To describe the class of GAMs, we let $q$ be a non-negative real number and $\eta:[0,1] \rightarrow[0,1]$ a continuous and strictly increasing function with $\eta(0)=0$ and $\eta(1)=1$. For each $q$ and $\eta$, 
the score distribution is the function $\phi_{q, \eta}: \mathscr{B}^{n} \times[0,1] \rightarrow[0,1]$ such that

$$
\phi_{q}(b, \eta, z)=\int_{0}^{z} \frac{s_{x}(b, q, \eta)}{\sum_{i=1}^{n} s\left(b_{i}, q, \eta\right)} d x .
$$

The Approval mechanism associates any profile $b$ with the alternative $\theta_{\alpha, q, \eta}(b)$ such that

$$
\theta_{\alpha, q, \eta}(b):=\min \left\{x \in[0,1] \mid \phi_{q}\left(b, \eta, \theta_{q, \eta}(b)\right)=\alpha\right\} .
$$

For any $b$ and any $i \in N$, we let $\psi_{-i}(b, q, \eta):=\sum_{j=1, j \neq i}^{n} s\left(b_{j}, q, \eta\right)$. Note that

$$
\phi_{q}(b, \eta, z)=\int_{0}^{z} \frac{s_{x}\left(b_{i}, q, \eta\right)+\sum_{j=1, j \neq i}^{n} s_{x}\left(b_{i}, q, \eta\right)}{s\left(b_{i}, q, \eta\right)+\psi_{-i}(b, q, \eta)} d x .
$$

with $s_{x}\left(b_{i}, q, \eta\right)=\frac{q}{\overline{b_{i}}-\underline{b_{i}}}+\eta^{\prime}(x)$ for any $x \in b_{i}$ and $s_{x}\left(b_{i}\right)=0$ otherwise, and $s\left(b_{i}, q, \eta\right)=q+\left(\eta\left(\overline{b_{i}}\right)-\right.$ $\left.\eta\left(\underline{b_{i}}\right)\right)$.

Since $\phi_{q}(b, \eta, z)$ is a score distribution, note that

$$
\sum_{j=1, j \neq i}^{n} s_{x}\left(b_{i}, q, \eta\right)<\psi_{-i}(b, q, \eta)
$$

for any $b, q, \eta$ and any $i \in N$. The next lemma shows how the outcome varies when a player varies the lower and upper bound of his strategy.

Lemma 3. Consider any profile $b=\left(b_{i}, b_{-i}\right)$ with convex support. Fix $\alpha, q, \eta$. Then, the GAM $\theta$ satisfies the following properties:

(1) if $\min b_{i}<\max b_{i}<\theta(b)$, then

$$
\frac{\partial}{\partial \min b_{i}} \theta\left(b_{i}, b_{-i}\right)>0 \text { and } \frac{\partial}{\partial \max b_{i}} \theta\left(b_{i}, b_{-i}\right)<0 .
$$

(2) if $\min b_{i}<\theta(b)<\max b_{i}$, then

$$
\frac{\partial}{\partial \min b_{i}} \theta\left(b_{i}, b_{-i}\right)>0 \text { and } \frac{\partial}{\partial \max b_{i}} \theta\left(b_{i}, b_{-i}\right)>0 .
$$

(3) if $\theta(b)<\min b_{i}<\max b_{i}$, then

$$
\frac{\partial}{\partial \min b_{i}} \theta\left(b_{i}, b_{-i}\right)<0 \text { and } \frac{\partial}{\partial \max b_{i}} \theta\left(b_{i}, b_{-i}\right)>0 \text {. }
$$

Proof. Consider first $\min b_{i}<\max b_{i}<\theta(b)$. Consider $x$ such that $F_{q, \eta}(b, x)=1 / 2$. Note that

$$
\frac{\partial}{\partial \max b_{i}} \phi_{q}(b, \eta, z)=\frac{\left.\left(\psi_{-i}(b, q, \eta)-\sum_{j=1, j \neq i}^{n} s_{x}\left(b_{i}, q, \eta\right)\right)\right) \eta^{\prime}\left(\max b_{i}\right)}{\left(\eta\left(\max b_{i}\right)-\eta\left(\min b_{i}\right)+\psi_{-i}(b, q, \eta)+q\right)^{2}}>0 .
$$

That is as $\max b_{i}$ increases $\theta(b)$ has to decrease for the outcome to be still equal to $\alpha$ so that $\frac{\partial}{\partial \max b_{i}} \theta\left(b_{i}, b_{-i}\right)<0$, as wanted. When we vary the lower bound of $b_{i}$, notice that

$$
\frac{\partial}{\partial \min b_{i}} \phi_{q}(b, \eta, z)=\frac{\left(\sum_{j=1, j \neq i}^{n} s_{x}\left(b_{i}, q, \eta\right)-\psi_{-i}(b, q, \eta)\right) \eta^{\prime}\left(\min b_{i}\right)}{\left(\eta\left(\max b_{i}\right)-\eta\left(\min b_{i}\right)+\psi_{-i}(b, q, \eta)+q\right)^{2}}<0 .
$$


Again, since $\min b_{i}$ increases $\theta(b)$ has to increase for the share of approvals to be still equal to $\alpha$ so that $\frac{\partial}{\partial \min b_{i}} \theta\left(b_{i}, b_{-i}\right)>0$, as wanted. The case in which $\theta(b)<\min b_{i}<\max b_{i}$ is symmetric and is omitted.

Consider now the case with $\min b_{i}<\theta(b)<\max b_{i}$. We can check that

$$
\frac{\partial}{\partial \max b_{i}}\left(\eta(x)-\eta\left(\min b_{i}\right)+q \frac{x-\min b_{i}}{\max b_{i}-\min b_{i}}+\sum_{j=1, j \neq i}^{n} s_{x}\left(b_{i}, q, \eta\right)\right)=\frac{q\left(\min b_{i}-x\right)}{\left(\min b_{i}-\max b_{i}\right)^{2}}<0
$$

whereas

$$
\frac{\partial}{\partial \max b_{i}}\left(\eta\left(\max b_{i}\right)-\left(\min b_{i}\right)+q+\psi_{-i}(b, q, \eta)\right)=\eta^{\prime}\left(\max b_{i}\right)>0
$$

Thus,

$$
\frac{\partial}{\partial \max b_{i}} \phi_{q}(b, \eta, z)<0,
$$

so that as $\max b_{i}$ increases $x$, has to increase. This shows that $\frac{\partial}{\partial \max b_{i}} \theta\left(b_{i}, b_{-i}\right)>0$, as wanted. Symmetrically, one can show that as $\min b_{i}$ increases $\theta(b)$ has to increase for $\phi_{q}(b, \eta, z)$ to be still equal to $\alpha$.

Q.E.D.

Once we have proved this key property of $G A M$, we prove that each $G A M$ satisfies the different properties used in the characterization.

Lemma 4. Any GAM satisfies IC.

Proof. Take some $b$ with $\theta(b) \neq t_{i}$ and $b_{i} \in S(\mathscr{B})$. Let $t_{i}<\theta(b)$ w.l.o.g. Applying Lemma 3 , it is simple to see that there always exists some $\delta>0$ such that $t_{i}<\theta\left(\left[t_{i}, t_{i}+\delta\right], b_{-i}\right)<\theta(b)$. Hence, there exists $c_{i} \in C(\mathscr{B})$ with $u_{i}\left(\theta\left(c_{i}, b_{-i}\right)\right)>u_{i}(\theta(b))$, as desired.

Q.E.D.

\section{Lemma 5. Any GAM satisfies MON.}

Proof. We now prove that for any GAM $\theta$, equivalence (1) holds. A similar proof applies to the characterization of the maximum of the attainable set.

1. Sufficiency. Take some $i \in N$ and assume that there is some $b_{i}^{*} \in \arg \min \theta\left(\mathscr{B}, b_{-i}\right)$ with $b_{i}^{*} \neq b_{i}^{m}$. Let $x^{*}=\theta\left(b_{i}^{*}, b_{-i}\right)$. Since $b_{i}^{*} \neq b_{i}^{m}$, this means that either $b_{i}^{*} \cap\left[0, x^{*}\right] \neq \emptyset$ (1.a.) or $b_{i}^{*} \cap\left[x^{*}, 1\right] \neq \emptyset(1 . \mathrm{b})$ or both (1.c). In each of these cases, Lemma 3 directly implies that $\theta\left(\left[0, x^{*}\right], b_{-i}\right)<\theta\left(b_{i}^{*}, b_{-i}\right)$, a contradiction with $b_{i}^{*} \in \arg \min \theta\left(\mathscr{B}, b_{-i}\right)$.

2. Necessity. Take some $i \in N$ and assume that there is some $b_{i}^{m}$ with $b_{i}^{m}=\left[0, x_{i}^{m}\right]$ and $x_{i}^{m}=\theta\left(\left[0, x^{m}\right], b_{-i}\right)$. Assume that $b_{i}^{m} \notin \arg \min \theta\left(\mathscr{B}, b_{-i}\right)$, so that there is some $b_{i}^{*}$ with $\theta\left(b_{i}^{*}, b_{-i}\right)<\theta\left(\left[0, x^{m}\right], b_{-i}\right)$. By definition, it must be the case that this means that either $b_{i}^{*} \cap$ $\left[0, x_{i}^{m}\right] \neq \emptyset$ or $b_{i}^{*} \cap\left[x_{i}^{m}, 1\right] \neq \emptyset$ or that both inequalities hold simultaneously. However, Lemma 3 again directly. proves that for any $b_{i}^{*} \in \mathscr{B}, \theta\left(b_{i}^{*}, b_{-i}\right) \geq \theta\left(\left[0, x^{m}\right], b_{-i}\right)$, which is a contradiction.

Q.E.D.

Lemma 6. Any GAM satisfies $C$. 
Proof. : Take any GAM $\theta$ with density function $f_{b}$. Take some $i \in N$ and any pair $b, b^{\varepsilon} \in B^{n}$ with $b^{\varepsilon}=\left(b_{i}^{\varepsilon}, b_{-i}\right)$ such that $\operatorname{Supp}(b), \operatorname{Supp}\left(b^{\varepsilon}\right) \in C(\mathscr{B})$. Assume moreover that $\lim _{\varepsilon \rightarrow \infty} b_{i}^{\varepsilon}=b_{i}$. We let $\phi_{q}(b, \eta, z)$ and $\phi_{q}\left(b^{\varepsilon}, \eta, z\right)$ respectively denote the score distribution associated to $b$ and $b^{\varepsilon}$. Since $\operatorname{Supp}(b), \operatorname{Supp}\left(b^{m}\right) \in C(\mathscr{B}), F_{b}(x)$ and $F_{b^{\varepsilon}}(x)$ are strictly increasing and continuous (hence invertible) on $\operatorname{Supp}(b)$ and $\operatorname{Supp}\left(b^{\varepsilon}\right)$. The respective inverse functions are denoted by $F_{b}^{-1}:[0,1] \rightarrow \operatorname{Supp}(b)$ and $F_{b^{\varepsilon}}^{-1}:[0,1] \rightarrow \operatorname{Supp}\left(b^{\varepsilon}\right)$. Therefore, $\lim _{\varepsilon \rightarrow \infty} F_{b^{\varepsilon}}^{-1}(x)=F_{b}^{-1}(x)$ for any $x \in \operatorname{Supp}(b)$. Since for any $b$ with invertible $F_{b}, \theta(b)=F_{b}^{-1}\left(\frac{1}{2}\right)$, it follows that $\lim _{\varepsilon \rightarrow \infty} \theta\left(b^{\varepsilon}\right)=$ $\theta(b)$, as wanted.

Q.E.D. 